\title{
A Theory of 'Auction as a Search' in Speculative Markets
}

\author{
Sudhanshu Pani
}

Saturday $4^{\text {th }}$ April, 2020

\begin{abstract}
The tatonnement process in high frequency order driven markets is modeled as a search by buyers for sellers and vice-versa. We propose a total order book model, comprising limit orders and latent orders, in the absence of a market maker. A zero intelligence approach of agents is employed using a diffusion-drift-reaction model, to explain the trading through continuous auctions (price and volume). The search (levy or brownian) for transaction price is the primary diffusion mechanism with other behavioural dynamics in the model inspired from foraging, chemotaxis and robotic search. Analytic and asymptotic analysis is provided for several scenarios and examples. Numerical simulation of the model extends our understanding of the relative performance between brownian, superdiffusive and ballistic search in the model.

Keywords: Market Microstructure, Levy Search, Limit order markets, Continuous Auctions, High Resolution, Zero Intelligence
\end{abstract}

MODERN MARKETS are built on the foundation of low latency trading and exchange infrastructure. Data available from the order driven markets today can help us to investigate the markets both in high frequency and high resolution. O'Hara (2015) called for the market microstructure toolbox to be enhanced in order to investigate 'orders' that were the unit of information in today's markets. Several recent papers implement objectives that require a high resolution analysis, including, modelling imperfect competition and quote cancellation (Baruch and Glosten (2019)), price discovery in high resolution (Hasbrouck (2019)), price discovery in quotes (Brogaard et al. (2019)), trading in absence of designated market maker (Kyle et al. (2018)). In the context of high resolution analysis, the assumption of equilibrium cannot be easily justified. However, such an assumption is fundamental to our current framework used to understand continuous double auctions in financial markets. In walrasian auctions in these markets, the price of a good is defined as the point where the supply and demand curves intersect (a market clearing equilibrium under perfect competition). The relationship between supply and demand and their linkage to price discovery may not however be effectively described by the assumption of walrasian equilibrium in high resolution of analysis. 
Even in quote driven markets, Beja and Hakansson (1977) had shown that the instantaneous walrasian adjustment imposes prohibitive demands on communication and computation, while the tatonnement iterations must take time, and thus it typically never converges. They further show that market making was necessary to price adjustments away from equilibrium. Modern order driven markets clear in the time scales that ranges from sub seconds to nano seconds. Donier and Bouchaud (2016) report the time required for walrasian adjustments to be in the scale of an hour. Jusselin et al. (2019) in their study based on Euronext exchange data and their model (presence of market maker and price discovery criterion) compute the optimal auction duration according to their criterion for 77 European stocks traded on Euronext. They report that the suggested durations are much larger than a few milliseconds, rather of order of 1 to 5 minutes. They reconfirm that continuous limit order book (CLOB) are in terms of their metric sub-optimal. However, the quality of the price formation process in CLOB market is not very far from that of the auction with optimal duration.

This paper explores 'Auction as a search' as an alternative to the supply and demand paradigm for tatonnement in order driven markets under the absence of an auctioneer or designated market maker. The questions we ask include: What is the modern day 'tatonnement' in the electronic order driven markets. Can we explain the dynamics of such markets as a 'search' by buyers for sellers and vice-versa.

The walrasian tatonnement is in effect an algorithm wherein an invisible auctioneer has all information regarding supply and demand schedules and comes up with the price that optimises the quantity traded. This emphasises an important function of the market, that of optimising the traded quantity. Any alternative algorithm needs to meet these two goals. We show in this paper that the tatonnement in continuous double auctions in modern asset markets under the usual price-time priority may indeed be described as a search of buyers by sellers and viceversa. 'Auction as a search' meets both the above stated objectives. An acceptance of this proposed alternative should help researchers, working on price discovery and trading models in high resolution, build models that are closer to reality.

\section{Introduction}

\subsection{Continuous Double Auctions}

The predominant market design used in asset markets around the world today is the continuous double auction mechanism (although these markets can vary in terms of the trading rules or matching rules they employ). This implies that an asset can theoretically be traded at any point in time. Double auctions enable all participants to provide quotes (both buy and/or sell orders). Futher these markets are organised as electronic exchanges, where 
the quotes from the traders are matched by matching engines without any official role for intermediaries. The matching is done everytime a new order comes into the market. Typically a mismatch may exist between buyers and sellers at any given instant. Hence, the need for an order based market with two fundamental kinds of orders. Traditionally, it was believed that impatient traders submited requests to buy or sell a given number of shares immediately at the best available price. Such orders are known as market orders. More patient traders submit limit orders, which also state a limit price, that correspond to the worst allowable price for the transaction. Limit orders may not result in an immediate transaction, and are stored in a queue called the continuous limit order book. Buy limit orders are termed bids, and sell limit orders offers or asks. Both limit orders and market orders play an important role in the order book. Institutional investors, large traders executing meta-orders and algorithmic traders deploy both limit orders and market orders to execute their trades.

A number of approaches have been used to model limit order markets. These include perfect rationality approaches, zero intelligence models and agent based models (refer Gould et al. (2013)). Zero-intelligence models, like the framework introduced by Bak et al. (1997), are useful to model the market activities as stochastic processes. Researchers have been able to reproduce a number of empirical regularities in the LOB in a zerointelligence framework using random walk diffusion models.

Given the continuous and sometimes sequential nature of the auctions, the walrasian model using supply and demand is the accepted solution to describe the equilibrium. Our contention in this paper is that when considering an analyis in high resolution we need to accept the existence of disequilibrium and look for an alternate mechanism for the emergence of prices and quantity trading.

\subsection{Random Walk to Levy Walk}

The concept of a random walk is a fundamental concept in finance used to model the stochastic evolution of the price of an asset. If $Y(Y \in R,-\infty<Y<\infty)$ is the log-price of an asset, $Y(t)$ represents the price at time $t(0 \leq t \leq \infty)$. The variance $\sigma^{2}(t)=\left\langle(Y(t)-\langle Y(t)\rangle)^{2}\right\rangle$ of the price series explains the dynamics of the asset price. (The brackets \langle\rangle denotes the mean. $\sigma$ is also known as Mean squared displacement (MSD) in diffusion literature). In Brownian motion, the variance varies linearly with time, $\sigma^{2} \propto(t)$. Prices in well functioning markets in the absence of arbitrage opportunities, are known to display brownian characteristics. A non linear time dependence of the variance may also be observed in the evolution of asset prices. Non linear time dependence is observed in several other complex systems. Such a non linear time dependence is a characteristic of anomalous diffusion, where $\sigma^{2}(t) \propto(t)^{\alpha}$, with $\alpha \neq 1$ (Denisov et al. (2012)). Anomalous diffusion has been observed in processes with long time correlations (such as the evolution of the transaction prices or the quoted prices for an asset). When 
$\alpha>1$, the case of superdiffusive limit, the random walker searches his environment much faster than 'Brownian search', provided the velocity is constrained to a limit so that he remains within the search space. The levy walk process is a simple stochastic model that combines these two notions - a superdiffusive evolution and finiteness of the velocity of motion.

The definition of the levy walk model is close to the random walk. A walker chooses a random direction and a random time $\tau$ and walks with a constant speed $v$ in the selected direction. After the time has elapsed a new random direction and a new random time are picked and the process repeats. What characterises levy walks is that, the duration of the walks are distributed according to a power-law density (probability density function, pdf): $\psi(\tau) \propto\left(\frac{\tau}{\tau_{0}}\right)^{-\gamma-1}$, where a constant $\tau_{0}$ sets the characteristic time scale and the exponent $\gamma$ determines explicitly the scaling of the corresponding MSD, namely $\alpha=1$ when $\gamma>2$ (normal diffusion), $\alpha=3-\gamma$ when $1<\gamma<2$ (superdiffusion), and the choice of the exponent from the interval $0<\gamma<1$ leads to the ballistic diffusion, $\alpha=2$.

The levy walk formalism has been successfully applied in diverse problems such as the description of DNA nucleotide patterns, modeling the dynamics of an ion placed into an optical lattice, analysis of the evolution of magnetic holes in ferrofluids and of photon statistics of blinking nanodots, engineering of levy glasses, an atom in an optical lattice, a tracer in a turbulent flow, T- cell motility in the brain, a predator hunting for food, or a mussel among a bunch of peers. (Denisov et al. (2012), Zaburdaev et al. (2015), Reynolds (2018)). Raposo et al. (2009) discuss the developments in random search process that includes levy formalism and random walks.

\subsection{Levy search in robotics and biology}

Levy search has been observed in biology (foraging and chemotaxis and others) and used extensively in robotics (a relatively nascent area of research). Foraging (the search for food) strategies of organisms have been used for thousands of years and optimised in this time period. Many of these search strategies (including chemotaxis) involve limited availability of information. The quest for efficient search algorithms has been influenced by behavioral science and ecology, where researchers try to identify the strategies used by living organisms. These provide motivation for our representation. In biology, levy walk is not confined to animal foraging and search. Movement patterns resembling levy walks have been observed at scales ranging from the microscopic to the ecological. They have been seen in the molecular machinery operating within cells during intracellular trafficking, in the movement patterns of T cells within the brain, in DNA, in some molluscs, insects, fish, birds and mammals, in the airborne flights of spores and seeds, and in the collective movements of some animal groups. Levy walks are also evident in trace fossils (ichnofossils) - the preserved form of tracks made by organisms that occupied ancient sea beds about 252-66 million years ago. And they are utilised by algae that originated around two billion years ago, and still 
exist today. (Reynolds (2018) and references therein). Existence of levy walk transport predate their formulation by researchers in the last thirty years. It provides motivation to model zero-intelligence search for survival using levy walk.

Autonomous mobile robots are required to explore the environment and locate a target. Targets could range from sources of chemical contamination to people needing assistance in a disaster area. Levy walks have recently emerged as universal search strategy in robotics. The finite velocity in levy walks that restricts search to the search region is useful in the context of robotics. The foraging trajectories of organisms look like periods of localized diffusive-like search activity altered with ballistic relocation to a new spot. This intuition is being explored in robotics. Performance evaluation of the robot or the search efficiency is another area where we draw ideas.

Our interest in levy walks as a search strategy emerges from the following: the speed of the search, the possibility to restrict the search space and the ability to work with limited information. These are the equipment that can work in high frequency double auction. We deploy a specific model of levy walks, ones where the velocities too are a random variable along with the flight time of the particles. This enables us to represent the behavioural dynamics of both the return expectations and time spent in the market by the traders.

In section 2, we propose in our model 'search' as a possible modern day tatonnement. The model deals with the total order book and is explained as a diffusion-advection-reaction process in Section 2.2 that also includes effects of imbalance between supply and demand. The diffusion part of the process is the focus in section 2.1 where in there is no imbalance between supply and demand. Diffusion is considered to be the main mechanism that can explain the price at which auctions are concluded. Several canonical models in finance have explained the evolution of price using Reaction-diffusion models and some of them extend to add jumps to the models (Brownian Semi-martingale or Brownian Semi-martingale jump process). When volume imbalances exist between buyers and sellers in the market, biases develop in the system and drift processes can emerge as additional dynamics to explain the price evolution and volume trading. Such an imbalance could be general imbalance or point imbalances at specific prices.

\section{SEARCH AS TATONNEMENT - The Total Order Book Model}

\subsection{The Search for trades in absence of bias}

We describe a model where buyers (buy orders) search for sellers (sell orders) and sellers search for buyers. This is a unique way of looking at predator-prey relationships (as either the buyer or seller could take the role of the predator or prey). Only when there is a bias in the system and an imbalance favouring either the buyer or seller, 
there will emerge a true predator-prey situation. We consider the total order book, comprising the visible part (the limit order book, LOB) and the hidden part (limit orders and market orders) that will be placed in the market in due course. The hidden part of the order book is also referred to as latent order book. We start with an LOB model based on the framework introduced by Bak et al. (1997), extend it to the total order book, TOB and later make appropriate changes to include behavioral dynamics of the agents. Orders are particles on a one dimensional lattice and their location corresponds to price (refer Fig 1). When orders representing a buy and sell occupy the same position on the one dimensional pricing grid it leads to a trade resulting into a reaction $A+B \rightarrow \emptyset$.

\subsubsection{The Agents}

We consider two types of agents in our model. The first can be characterised as noise traders with zero intelligence. The noise traders start their journey by placing market orders at the last transaction price. However, in the model they are not required to absorb losses. They are zero intelligent because once they transact through a market buy (sell) order, they place a subsequent limit sell (buy) order to occupy the price at the best offer (bid). In the simulation of the model (Section 3), for example, we implement this by placing a limit offer at 0.1 USD (an arbitrary choice) over the transaction price they record as a market order or a limit buy at 0.1 USD lower than the transaction price. Once such a limit order is transacted they can again go back to place market order and the cycle repeats. The endevour is to remain in the market and get involved in as many transactions.

The second type of agents are the strategic traders. These agents represent a heterogenous group of investors who have a view on future transaction prices. Or they may have specific portfolio execution mandates. They seek specific prices to enter into transactions. The origin of pricing decision (as well as quoted volume) may be varied: portfolio decisions, private information, liquidity concerns, meta-order execution, fundamental value etc. Strategic traders may be buyers or sellers and use limit orders or market orders to execute their transactions. When they place market orders, they come into the market at the specific price and expect execution of the trade. They leave the market after an execution.

\section{Order cancellation by agents}

Cancellation of orders sets up useful behavioural dynamics in these markets. Benzaquen and Bouchaud (2018) use cancellations to model the supply and demand in the order book. In section 2.1, neither the noise traders nor the strategic traders come back to the market in case orders are cancelled before execution. Quotes from noise traders are cancelled in section 2.1 and 2.2 under two circumstances. First, when the particles end their designated flight time and second in case the available budget with the trader falls short of a limit ask order. We treat it as 


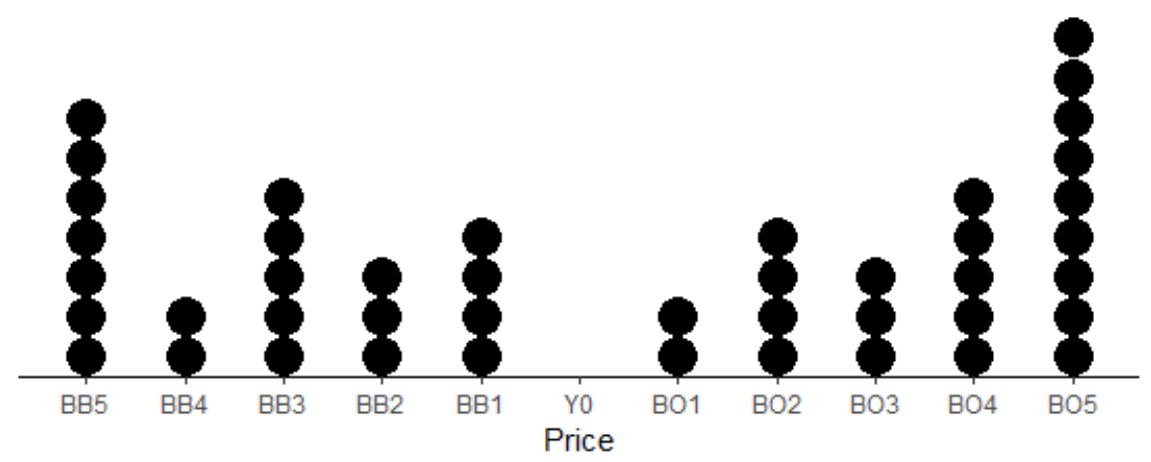

Figure 1: One dimensional lattice showing price and orders shown as particles stacked up at the price. If a buy and sell particle occupy a single lattice point it leads to a transaction. Y0 is the last transaction price and does not have any market particles.

a default in the trading process and remove the quote and trader. Quotes from strategic traders are cancelled in both section 2.1 and 2.2 when they end their flight time. Additionally, in the examples in section 2.2 to illustrate the traders response to biased trading conditions, we allow orders from strategic traders to be cancelled and a new order placed again. These are quote revisions.

The merit of our model is to show that in high resolution price discovery and trading of volume in financial markets can be achieved using a model of search.

Consider a continuous auction, order driven market, in a relatively homogeneous state. A homogeneous state in the order book can be achieved by a constant gradient or uniform gradient in the quantity density. The equivalent in economics perspective would be that the demand and supply is balanced. We want to avoid perturbations due to larger buy or sell orders causing bias in the system. We envisage a model where market buy (or sell) orders search for targets - sell (or buy) orders, to effect a trade. The target could be a market order or limit order. A market order is one where a transaction can get effected immediately if a counterparty is available. The behaviour of the agents represented in the form of orders is treated as particles. One may consider a single particle to be equivalent to the smallest quantity of the order that is allowed to be placed in the market.

\section{The Total Order Book}

The total order book comprises the limit order book (visible LOB) and the latent orders. Latent orders are orders that are yet to be placed in the market, but the traders have already planned the same. These include the limit orders that would be placed in future and the market orders. Our conceptualisation is not based on price but on the 


\section{REPRESENTATION OF THE TOTAL ORDER BOOK}

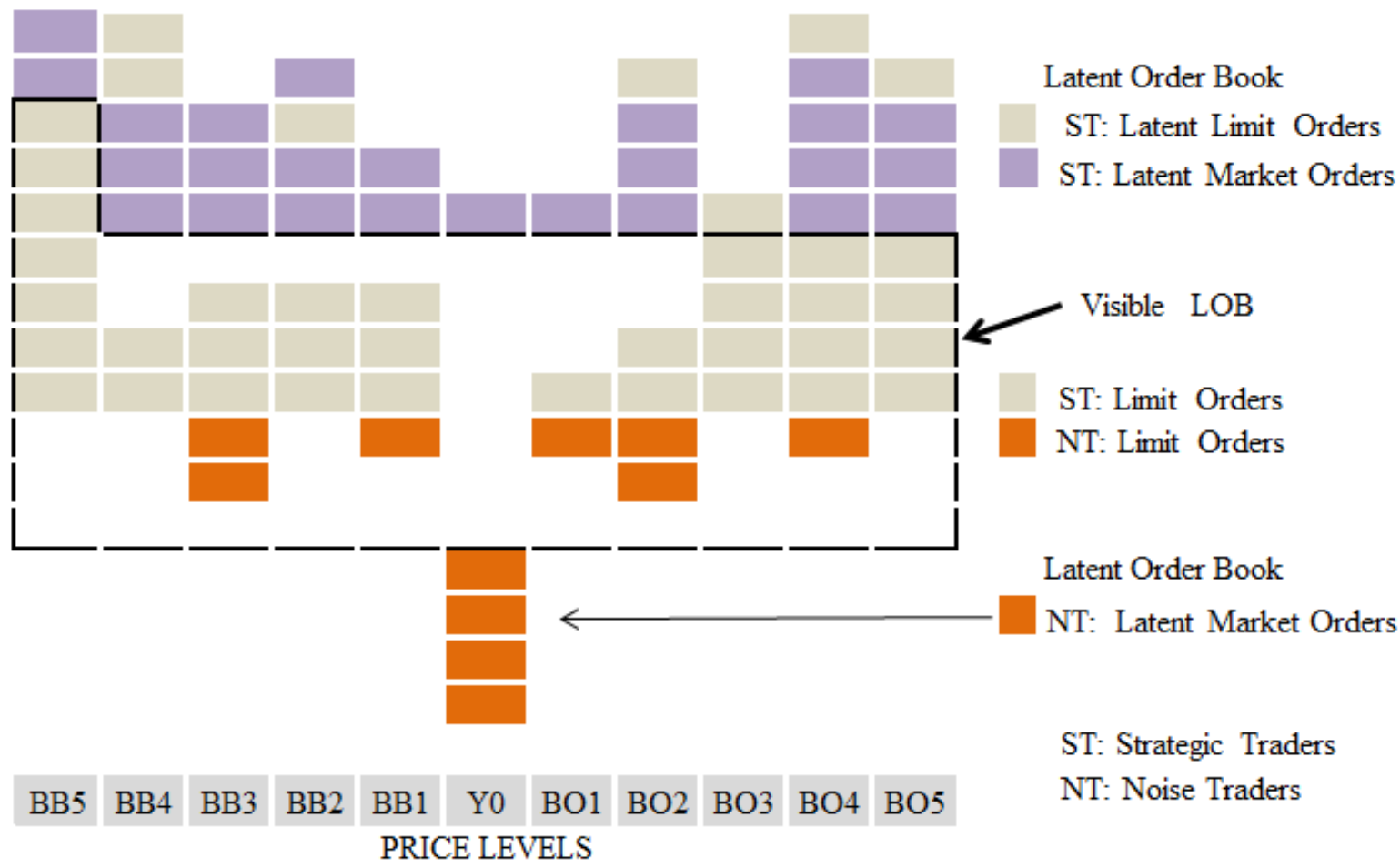

Figure 2: Representation of the Total Order Book as a one dimensional lattice. It comprises the visible limit order book and the latent order book. The dark border represents the visible limit order book given in fig 1 . The limit orders from strategic traders (ST) are in grey. The limit orders from noise traders (NT) are placed immediately after a market order is executed by a NT. Strategic traders can place limit orders (grey) or market orders (purple) in future and hold their orders in the latent order book. Noise traders place market orders at the last transaction price Y0. BO-Best Offer; BB- Best Bid.

quoting strategy of the agents. Thus when orders are present in the latent order book, the traders have decided on the price, time, sequence or events at which they would introduce the orders into the visible order book. A market order in the latent (hidden) order book is introduced as a market order necessarily at specific predecided price, time or sequence. The order does not carry a price, but is only introduced when the transaction price is around the predecided price. A limit order in the latent book, in contrast, will carry a limit price and would be introduced by the traders into the visible LOB at a predecided time, sequence or event. Such a representation addresses the practice of algorithmic trading and slicing of metaorders into child orders.

Fig 2 represents the total order book. It super imposes the strategic and noise particles into the limit order book model depicted in fig 1 .

To investigate the tatonnement process, we note that any transaction necessarily requires a market order as atleast one of the counter party. We suggest that modelling the market orders should be able to explain all trans- 


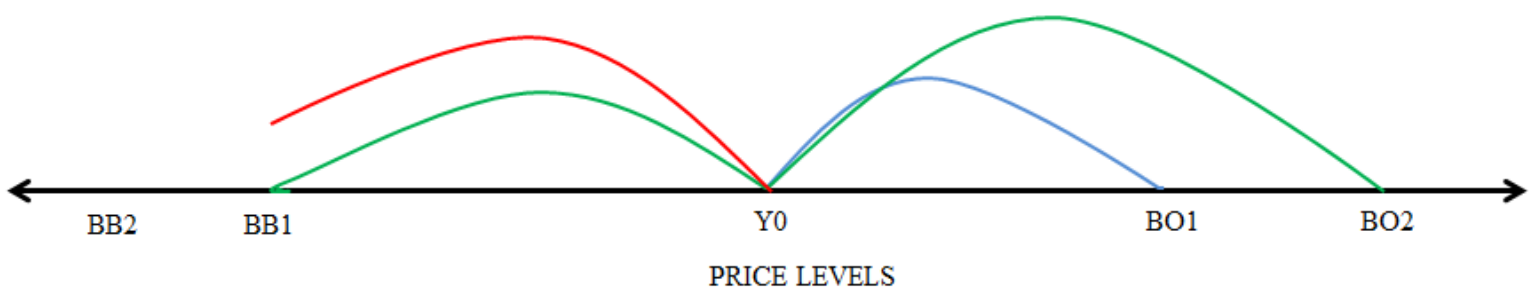

Figure 3: Levy Search. The fate of 4 market orders particles introduced at the last transaction price Y0 is represented. These particles are introduced sequentially starting time $t_{0}$ after the last transaction occurs. The particles differ in the velocity imparted to them and time spent in the market before cancellation. Both these variables are random variables in the model. The sign of the velocity gives the direction of flight and represents a buy order or a sell order. The market orders can be placed by both noise traders and strategic traders. The next transaction occurs when the first particle reaches the target at time $t_{1}$, in this example, the best offer BO1, which is assumed to have a limit order. At this instant three particles are still in flight. Two market particles reach targets at BB1 (time $t_{2}$ ) and BO2 (time $t_{3}$ )and are transacted sequentially. One particle is cancelled before it can reach the target. Between time $t_{0}$ and $t_{3}$ many more orders may have been introduced and it is not represented in the figure.

actions and help us achieve our objective. The reduction in the dimensionality is an important benefit.

Let $u(x, t)$ be the probability density function (pdf) of the distance travelled by market order particles in search of trades. Thus, $x$ is the total length of the jumps and $t$ is the total time. We can represent $u$ as a continuous time random walk. Let, $Y_{0}$ be the last traded price and $Y_{1}$ be the next expected transaction price. When $Y_{0}$ occurs, it ends the flight of some of the market order particles (those that have reached or crossed the price $Y_{0}$ ). However, some particles are still in flight. Between, $Y_{0}$ and $Y_{1}$, a number of new quotes are placed into the book, some particles complete their flights through cancellations and at least one would complete their flights with the transaction ending at $Y_{1}$. We consider particles that have completed their search with success or ended with cancellation. (refer Fig. 3 where $Y_{0}=Y 0$ and $Y_{1}=B O 1$ ).

We first specify the price quoting and cancellation behaviour and then we shall resume with the model.

Quoted Price and Cancellation Traders display their intentions by placing market and limit orders. They impart a velocity $v$ to the particles and the orders have a flight time $\tau$. The flight ends with a quote cancellation or trade and the particles are believed to be at rest before entering the market. The particles enter the market either into the LOB or into the latent order book as limit or market orders. The length of the walk or jump of a particle can be represented by the coupling $v \tau$. This is close to the model in Zaburdaev et al. (2008) that dealt with a 
random walk with random velocities.

The velocity imparted to the orders from strategic traders depends on certain dynamics experienced by the traders and is an important element that determines the quoted price. For any trader, it is governed by his expectation of success. The strategic traders have specific prices that they want to quote. This price may also be viewed relative to the return they desire from the first transaction price of the trading day. Let the expectation of this return for the $j t h$ trader be $\left\langle r_{j}\right\rangle$ and the averages of the velocity and flight time be denoted by $\bar{v}_{j}$ and $\bar{\tau}_{j}$ respectively. So, the following holds:

$$
\left\langle r_{j}\right\rangle=\bar{v}_{j} \bar{\tau}_{j}
$$

The above dynamics is guided by the past experience of success, current trading conditions and state of the order book. We do not investigate these dynamics and to simplify, we treat the velocity and flight times as random variables drawn from distribution. The velocity imparted along with the flight time determines the quoted price. The strategic traders move out of the market after a trade execution or at the end of the flight time.

Noise traders quote the last transaction price in their market orders and a small profit over the last transaction price while quoting limit orders. This over-rules any role of velocity. The flight time in case of quotes from noise traders determines when any quote from the noise trader is cancelled and the trader moves out of the market.

The particle is assumed to be in-flight if its current price (last transaction price after which it entered the market + the jump) is still less than the transaction price quoted and it is not cancelled. The velocity $v$ can have positive and negative values to include buy or sell orders (direction of motion). The inter trade time period is known as a trade duration. If ' $N$ ' number of trades takes place in a unit of time $d t$, the average duration can be given by $\langle\mu\rangle=d t / N$. Each inter-trade period is a search for the next transaction. A number of jumps get recorded in this period.

Equation (2) gives the total path traversed in the search $x$ till time $t$. Driven by return expectation in (1) the quote from a trader is provided with a velocity drawn from a pdf $h(v)$ and the flight time (cancellation behaviour) from a pdf $f(\tau)$. The distance travelled by such a particle during search is given by the coupling $v \tau$.

Flight time and velocity are the two basic and independent random variables of the model. They are normalised to $1, \int_{-\infty}^{\infty} h(v) d v=1$ and $\int_{0}^{\infty} f(\tau) d \tau=1$. In the base case, to avoid a bias in the system, the velocity distribution is symmetric, $h(v)=h(-v)$. However, interactions due to limit orders or transactions in the system can change this assumption and we shall model this in section 2.2.

$$
u(x, t)=\int_{-\infty}^{\infty} d v \int_{0}^{t} u(x-v \tau, t-\tau) h(v) f(\tau) d \tau+n_{0}(x) \delta(t)
$$


The right side in (2) describes the dynamics of search where the distance of $v \tau$ is traveresed in time $\tau$ so that the total distance traversed in search reaches $x$ from $x-v \tau$ and total time spent in search reaches $t$ from $t-\tau$. Taking into account the possible velocity-time coupling, the joint probability $h(v) f(\tau)$, the first term in (2) integrates over all flight time and velocity combinations. Since a coupling of velocity and time represents an order, it in effect integrates the distance travelled for all search orders. For particles that have completed their flights, they would have reached a point on the lattice grid. It may or may not have resulted in a success. For all such flight times, we sum the total distance travelled or jumps for all velocities. Particles still in flight are not included. The initial distribution of the market particles is given by $n_{0}(x)$. This search is the tatonnement in modern markets.

The success of each search is a trade transaction. The pdf of the traded particles, $q(x, t)$ is simply the number of trades that take place in a time interval $(0, t)$. However, the trades being the success of the search of market particles in a tatonnement, we define the efficiency $\eta$ of the search process as the ratio of the total path traversed in the search to the number of targets searched or trades done. This gives us a link to represent $q(x, t)$ in terms of $u(x, t)$ and $\eta$ as in equation (3). $\eta$ is again a function of the coupling of velocity imparted and the flight times.

$$
q(x, t)=\int_{-\infty}^{\infty} d v \int_{0}^{t} \frac{u(x-v \tau, t-\tau) h(v) f(\tau)}{\eta(v \tau)} d \tau
$$

Equations (2) and (3) fully describe the dynamics of the system with a given initial density of particles and the two pdf for the flight times and velocities. They establish the crucial link between the tatonnement in the auction with the trades. Next we solve these equations analytically. First, we determine the total path traversed by market particles in the search process and then introduce the result into the equation of the trade density. We apply the Fourier transform with respect to the spatial coordinate in (2) and then the Laplace transform with respect to time. This yields in (4) the path traversed in search in the Fourier-Laplace domain, $k, s$, as:

$$
u_{k, s}=\frac{n_{0, k}}{1-\left[h_{k \tau} f(\tau)\right]_{s}}
$$

We can introduce the result in (4) into the Fourier-Laplace expression for equation (3) to get the analytic expression for the density of trades in the Fourier-Laplace domain, obtained through the search process in (5).

$$
q_{k, s}=\frac{n_{0, k}}{\eta_{k, s}} \frac{\left[h_{k \tau} f(\tau)\right]_{s}}{\left(1-\left[h_{k \tau} f(\tau)\right]_{s}\right)}
$$

To find a solution, the next step is to take the Laplace inverse of equation (5). However, an analytic representation and direct inversion of the equation (5) is not feasible. Froemberg et al. (2015) recommend an asymptotic analysis for large space and time scales, $x, t \rightarrow \infty$. We use the same approach. Going to Fourier-laplace space 
using the tauberian theorem, this limit corresponds to $(k, s) \rightarrow(0,0)$ such that $k / s=$ constant. This has to be performed numerically.

It is important to define the velocity and flight time distributions before we move to obtain the inverse transformation. In our view this needs to come from empirical analysis. Further, velocity distribution cannot be obtained directly as it is a notional quantity and needs to be interpreted from equation (1) that describes the relationship with returns.

As discussed in Zaburdaev et al. (2008), Froemberg et al. (2015) and Zaburdaev et al. (2015) when the velocity distribution is Cauchy or lorentian, the density of the particles also is lorentian, independent of flight times and jump lengths. Such a lorentian velocity profile appears in real physical phenomena such as two dimensional turbulence and is also found in model distributions of kinetic theory, statistics, plasma physics and starving amoeba cells. We know that a cautchy process does not give rise to a continuous sample path for the price and it differs from Brownian motion as there are large jumps not infrequently. As given below, we make arbitrary choice of a lorentian velocity distribution $h(v)$ and intuitively a flight time distribution $f(\tau)$ with power tails.

$$
\begin{gathered}
h(v)=\frac{1}{u_{0} \pi} \frac{1}{\left(1+\left(\frac{v^{2}}{u_{0}^{2}}\right)\right)} \\
f(\tau)=\frac{\gamma}{(1+\tau)^{1+\gamma}}
\end{gathered}
$$

In equation (6) $u_{0}$ is needed to constrain the velocities so that the particles do not go beyond the ballistic cones, else it will lead to instantaneous dispersion. The $\gamma$ in equation (7) is varied to get different transport. In equation (5), given the asymptotic limit we want to evaluate, we further set a constant efficiency, so that there is an expression for the initial density of particles for the trade density. The propagator for our model can then be noted as follows:

$$
G(k, s)=\frac{\mathcal{L}[f(\tau) h(k \tau)]}{1-\mathcal{L}[f(\tau) h(k \tau)]}
$$

where $k \tau$ is the Fourier variable conjugate to $v$. The equation (8) retains the form of the well known MontrollWeiss equation for the pdf of the uncoupled continuous time random walk (CTRW) to find the particle $x$ at the time $t$, modified such that it applies to random jumps in velocity. Equation (8) can be rewritten as (see Appendix C for details):

$$
G(k, s)=\frac{\left.\int_{-\infty}^{\infty} d v f(s+i k v \tau) h(v)\right]}{\left.1-\int_{-\infty}^{\infty} d v f(s+i k v \tau) h(v)\right]}
$$


For the flight time distribution we have chosen and in the long time limit the expansion in the Laplace space is given by,

$$
f(\tau) \approx 1-\tau^{\gamma} \Gamma(1-\gamma) s^{\gamma}
$$

Using (10) the asymptotic version of (9) is,

$$
G(k, s)=\frac{1}{s} \frac{\int_{-\infty}^{\infty}(1+i k v / s)^{\gamma-1} h(v) d v}{\int_{-\infty}^{\infty}(1+i k v / s)^{\gamma} h(v) d v}
$$

\section{Random walk with Ballistic scaling}

As the simulation in section 3 confirms, ballistic scaling may be useful in thinly traded stocks where the arrival rates of orders, transactions and cancellations is low. A method exists for the inversion of the fourier laplace expression for propagators with ballistic scaling. A propagator of a random walk model has ballistic scaling if it can be written in the form: $G(x, t) \approx \frac{1}{t} \phi\left(\frac{x}{t}\right), t \rightarrow \infty$, where $\phi$ is the scaling function. In Fourier-laplace space this is, $G(k, s) \approx \frac{1}{s} g\left(\frac{i k}{s}\right)$. Comparing the two forms we can rewrite the scaling form of our equation as in equation (12), where $\xi=\frac{i k}{s}$.

$$
g(\xi)=\frac{\int_{-\infty}^{\infty}(1+\xi v)^{\gamma-1} h(v) d v}{\int_{-\infty}^{\infty}(1+\xi v)^{\gamma} h(v) d v}
$$

Using a arbitrarily chosen special situation induced by Cauchy distributed velocity and taking the inverse Laplace and Fourier transform we get a form of cauchy distribution as in (13) (refer Appendix B).

$$
G(x, t)=\frac{u_{0} t}{\pi\left(u_{0}^{2} t^{2}+x^{2}\right)}
$$

Although $u_{0}$ comes in due to the need to restrict the particles in the ballistic cone, it is an important feature as it slows down the diffusion process. The density of trades scales inversely with time and square of the price.

\section{Role of diffusive search and the traders}

The role of diffusive levy search is to provide continuity in the auction and also volume trading. The nature of this continuity can be evaluated basis the success of the search in terms of the density of trades. This is further related to the inter trade duration. The parameters that determine the trade density are thus critical to continuous auction. First, the presence of noise traders and the market orders placed by noise traders and strategic traders. Market orders need to be always available to provide continuity in auctions. Else, traders need to cancel and revise their quotes, which we shall discuss in section 2.2. Trading can slow down if less number of noise traders are present or if they get locked away post transaction either in limit orders or leave the market. We always expect strategic 
traders to be present in the market. But presence of noise traders can be impacted by market design. Second, the efficiency of the search. The efficiency is dependent on the resource density or target density. This comprises both of the limit orders and strategic market orders that come in whenever a new price is discovered. It is these market orders that bring in efficiency improvement by leading to greater number of transactions at already discovered price.

\section{Efficiency of Search}

A useful tool to judge how a trading system is performing and in the case of this model, how the search for trades performs is to evaluate a global efficiency of the search using the trade duration. The trade durations is the time diference between one trade and the next. Thus it is the time between any two auctions in continuous trading set up. The reciprocal of the trade duration is rate of trades. If the searchers are taking longer flight times or longer distance in the walk before they locate the target, the rate of trades would decrease. This measure is an indirect route to use the concepts like distance travelled per success or average flight time per success, but is more meaningful and easier to evaluate for a trading system. The rate of trades or efficiency of search can be computed as $\left\langle\frac{1}{\tau}\right\rangle$, where $\tau$ is the trade duration. A similar measure for efficiency of search in a different context was also used by Palyulina et al. (2014). In section 3 (numerical simulation) we have used this measure (rate of trades) as the efficiency of search to compare search in ballistic, superdiffusive and brownian regimes with different market conditions.

\section{Need for velocity as random variable}

The use of Random velocities in the modelling is unique in the context of modelling stock prices. It is not required when modelling the trade prices, but required when modelling the quotes. As discussed earlier, the velocities are a notional quantity and cannot be directly observed. This technique is a useful tool to connect the random walks of quotes to the trade price series. Most quotes are cancelled sooner or later and hence do not get involved in the reaction. The random velocities through the return expectations can connect the trade durations to the quote durations.

\subsection{The Complete Model - Search for trades in presence of bias}

In section 2.1 we dealt with a limit order market (Total Order Book) in homogeneous state where the search is diffusive. A situation where there is no shortage of noise traders and strategic traders have placed market orders at widely dispersed prices. The diffusive search was crucial to explain price continuity. This section combines 
diffusive search with other mechanism that can explain auctions when we relax the assumption of 'no bias'. In case of excess demand (buy or sell side) of liquidity a bias will get generated in the system, resulting in a drift. The market will move to improve the search efficiency due to higher availability of resource targets, i.e more and more targets can be acquired at the existing transaction price. The number of targets thus go up without increase in the path travelled in the search leading to higher efficiency. The market will naturally drift from resource rich regions to regions where market order particles may be available. This is because market orders from strategic traders are only placed when the relevant prices and time is reached.

Further, there is a stochastic evolution of the transaction price of the asset and quantities traded. This is the result of the strategic games between the market orders and limit orders, buyers and sellers, new order arrivals, order cancellations and transactions. We allow for such a stochastic evolution although we model the dynamics of market order particles only. Since market order are involved with each transaction, we expect to successfully explain the stochastic evolution of the transaction prices and volume traded. Donier et al. (2015) use a diffusionadvection-reaction model to explain the evolution of the marginal supply and demand in the market. We set up a model where market order particles search for targets in the double auction market. While we classify our model as a diffusion-advection-reaction model, given the view point of search, it has been built using concepts involving foraging, predator-prey search and chemotaxis (Grunbaum (1998) and references there in).

In section 2.1, $Y\left(Y \in R^{+}, 0<Y<\infty\right)$ represented the price of an asset and $Y(t)$ the price at time $t(0 \leq t \leq \infty)$. We denote the price by $x$ in this section (in section $2.1 x$ denoted total distance travelled).

Let $g(x, t)$ represent the density of market particles at a price $x\left(x \in R^{+}, 0<x<\infty\right)$ and time $t(0 \leq t \leq \infty)$. Our fundamental set up is given by the partial differential equation (14).

$$
\begin{gathered}
\frac{\partial g(x, t)}{\partial t}= \\
\frac{\partial}{\partial x}\left(D(t) \frac{\partial g(x, t)}{\partial x}-\lambda_{1}(t) \frac{\partial f(x, t)}{\partial x} g(x, t)-\lambda_{2}(t) g(x, t)\right)+v(t) g(x, t)+q(x, t)
\end{gathered}
$$

\section{The set up in equation (14)}

(14) explains the stochastic time evolution of the density of market order particles in our one dimensional grid. The first term on the right hand side represents diffusion that we discussed in section 2.1. The second and third terms relate to volume trading and price evolution due to drift. The fourth term is the net addition of market particles. The last term represents large external additions of market particles into the system.

Section 2.1 can explain a walrasian tatonnement where prices are set when the quantity demanded by buyers matches the quantity supplied by sellers. Modern order driven markets operate at a speed where there is neither 
sufficient time to establish a walrasian equilibrium nor there is an official market maker who will adjust the price instantaneously. Hence, transactions take place at prices out of equilibrium. Traders have always observed markets operating out of equilibrium. Even in Quote driven markets as noted by Beja and Hakansson (1977) and Beja and Goldman (1980), set up of equilibrium needs more time to allow for the information flow required for the purpose. We look at (14) as the mechanism that can take the trading system towards equilibrium. We now explain how the terms enter the right hand side in (14) :

- Diffusion (first term): This is the primary search mechanism through which price continuity and quantity transport takes place. In the absence of bias this will remain the only mechanism (already discussed in the Section 2.1).

- Drift due to target density (second term): We implement a formulation widely used in foraging studies ( Grunbaum (1998)). In the set up of those studies, the drift velocity is proportional to the gradient resource density. And the coefficient is the taxis coefficient. The use of resource density implies the dimensionality of the model increases. We, however, note that the density of targets (limit orders or market orders) in our use case should result either into a response or consequence in the system. We model the drift resulting from this response or consequence in market particles. $f(x, t)$ is the perceived resource density basis the actions in the system. In this innovation we conjecture that the drift resulting from a gradient of resource density should have a mirror image in the searcher. This mirror image may be different than the original gradient, but is more relevant as it is likely to be a tactical response in the absence of complete information (since the visible order book is not the complete order book). We may err in magnitude but not direction and the system will try to iteratively guess the true gradient of resource density. Again we accomodate a time evolving coefficient $\lambda_{1}$.

- Drift due to movement within the market order particles (third term): There are several scenarios we envisage where a number of market particles could get activated into directed activity resulting in a drift. For example, high transaction activity on one side of the market, will result in noise traders getting released from limit orders suddenly due to the reactions. This early release increases returns for noise traders. If these traders sense the action to continue, they would rush to the other end to repeat the cycle. Similarly, traders yet to place orders may anticipate change in resource gradient and resource density due to news flow and react by adjusting their own positions. $\lambda_{2}$ is a time varying coefficient .

- Net addition to market particles density $v$ (fourth term) : $v(t)$ is a time varying composite coefficient of the new order additions $v_{a}$, cancellations $v_{c}$ and transactions (reactions) $v_{r} . \quad v(t) g(x, t)=v_{a}(t) g(x, t)-$ 
$v_{c}(t) g(x, t)-v_{r}(t) g(x, t)$. New order additions of market particles includes market particles, hitherto latent, coming into the order book, new traders, limit orders that are cancelled and placed as market orders as the traders become impatient. Hence, this can be a non-trivial component. Cancellations and transactions remove market particles of strategic traders from the order book. Noise traders move out of market only at end of their flight time.

- $q$ (fifth term) is a source of market particles much larger than what comes in during the average trading day for the asset. It could be a demand or a supply of liquidity. The source may be a single or few point sources or distributed across a price range and even in time.

In summary, we build a comprehensive model of stochastic evolution of market particles. Expand (14) to obtain (15) :

$$
\begin{gathered}
\frac{\partial g(x, t)}{\partial t}= \\
D(t) \frac{\partial^{2} g(x, t)}{\partial x^{2}}-\lambda_{1}(t) \frac{\partial f(x, t)}{\partial x} \frac{\partial g(x, t)}{\partial x}-\lambda_{1}(t) \frac{\partial^{2} f(x, t)}{\partial x^{2}} g(x, t) \\
-\lambda_{2}(t) \frac{\partial g(x, t)}{\partial x}+v(t) g(x, t)+q(x, t)
\end{gathered}
$$

Equation (15) can be solved analytically. We draw upon the technique used by Sanskrityayn and Kumar (2016), who used the Greens function method to solve their diffusion-advection equation in the context of pollutant solutes in the atmosphere. To solve the equation, we note that $q$ will remain untouched and we need to reduce the equation to a known form so that we find an expression for $f(x, t)$. We do a co-ordinate transformation from the domain $(x, t)$ to the domain $\left(X(x, t), t^{\prime}\right)$. The domain $\mathrm{X}$ is essentially fixed time-snapshots of the entire lattice. We want to transform (15) to the form in equation (16).

$$
\left.\frac{\partial G\left(X, t^{\prime}\right)}{\partial t^{\prime}}=D_{1}\left(t^{\prime}\right) \frac{\partial^{2} G\left(X, t^{\prime}\right)}{\partial X^{2}}-\lambda\left(t^{\prime}\right) \frac{\partial G\left(X, t^{\prime}\right)}{\partial X}+v_{1}\left(t^{\prime}\right) G\left(X, t^{\prime}\right)\right)+q_{1}\left(X, t^{\prime}\right)
$$

Using the domain transformation, we can write equation (15) as equation (16), following which we equate the coefficients to solve for the interim variables and transformations introduced. (Refer Appendix D for details). Using the transformations we can reduce our initial equation to the following form,

$$
\frac{\partial G(X, t)}{\partial t}=\frac{D(t)}{\beta^{2}(t)} \frac{\partial^{2} G(X, t)}{\partial X^{2}}-\lambda(t) \frac{\partial G(X, t)}{\partial X}+v_{1} G(X, t)+q_{1}(X, t)
$$


where the dimensionless expressions and transformation are given in (18), (19) and (20).

$$
\begin{gathered}
\frac{D_{1}(t)}{D(t)}=\frac{1}{\beta^{2}(t)} \\
\beta=e^{\int_{0}^{t}\left(v(s)-v_{1}(s)\right) d s} \\
X=\frac{x}{\beta(t)}+\int_{0}^{t}\left(\lambda(t)-\frac{\lambda_{2}(t)}{\beta(t)}-\lambda_{2}(t) \frac{\phi_{2}(t)}{\beta(t)}\right)
\end{gathered}
$$

The initial conditions for this equation are $G(X, 0)=G_{i} \omega(X)$, with $-\infty<X<\infty$ and $t>0$. Next, we try to remove the drift term and the decay term from (17) by using further transformations. We use the following transformation equations one after the other for this purpose. In (22) $\beta_{1}=e^{\int_{0}^{t} v_{1}(s) d s}$ is a dimensionless term and in (23) $\beta$ is as per (19). In (23) $T$ is a time variable. Equation (17) now reduces to (24).

$$
\begin{gathered}
\eta=X-\lambda(t) t \\
K(\eta, t)=\frac{G(\eta, t)}{\beta_{1}(t)} \\
T=\int_{0}^{t} \frac{1}{\beta^{2}(s)} d s \\
\frac{\partial K(\eta, T)}{\partial T}=D \frac{\partial^{2} K(\eta, T)}{\partial \eta^{2}}+\frac{Q(\eta, T) \beta^{2}(T)}{\beta_{1}}
\end{gathered}
$$

We now need to solve equation (24) to obtain a master equation for transport of market particles in double auction limit order asset markets. Haberman (1987) provides a solution for equations such as (24) using Greens Function Method (GFM). The solution to (24) based on GFM is given in (25):

$$
\begin{aligned}
K(\eta, T) & =\int_{0}^{T} \int_{-\infty}^{\infty} \frac{Q(\chi, \tau) \beta^{2}(\zeta)}{\sqrt{4 \pi D(T-\zeta)} \beta_{1}} \exp \left(-\frac{(\eta-\chi)^{2}}{4 D(T-\zeta)}\right) d \chi d \zeta \\
& +\int_{-\infty}^{\infty} \frac{1}{\sqrt{4 \pi D T}} G_{i} \omega(X) \exp \left(-\frac{(\eta-\chi)^{2}}{4 D T}\right) d \chi
\end{aligned}
$$

Next we sequentially trace back the transformations done earlier, in reverse order to get the solution below in 
(26). Here, $\zeta=\int_{0}^{\tau} \frac{1}{\beta^{2}(s)} d s$ and the initial condition $g(x, 0)=G_{i} \omega(x)$.

$$
\begin{gathered}
g(x, t)=\beta_{1}(t) \int_{0}^{t} \int_{-\infty}^{\infty} \frac{Q(\chi, \tau)}{\sqrt{4 \pi D(T-\zeta)}} \exp \left(\frac{\left(\frac{x}{\beta}-\int_{0}^{t}\left(\frac{\lambda_{2}}{\beta}+\frac{\lambda_{1} \phi_{2}}{\beta}\right) d s-\chi\right)^{2}}{4 D(T-\zeta)}\right) d \chi d \tau \\
+\beta_{1}(t) \int_{-\infty}^{\infty} \frac{1}{\sqrt{4 \pi D T}} G_{i} \omega(\chi) \exp \left(-\frac{\left(\frac{x}{\beta}-\int_{0}^{t}\left(\frac{\lambda_{2}}{\beta}+\frac{\lambda_{1} \phi_{2}}{\beta}\right) d s-\chi\right)^{2}}{4 D T}\right) d \chi
\end{gathered}
$$

Before we move into asymptotic analysis, a few observations are due:

\section{The significance of $\beta$.}

The dimensionless quantity $\beta$ has a physical meaning and is not simply an artefact of domain transformation. On transforming the domain from $(x, t)$ to $\left(X, t^{\prime}\right)$, each point on the one dimensional lattice is now a space-time variable. The advantage of this construction is that $X$ alone can explain the time evolution of the system. We have used this intuition to our advantage. $\beta$ relates the diffusion in the new domain to the original domain in (18). Should the diffusion coefficient be different in the two domains, since we have defined diffusion coefficient as a variable only in time. Since, $\beta$ is defined in (19) in terms of the relative contribution from the net addition to the density of market particles in the two domains, we infer that at any particular price in the domain $\left(X, t^{\prime}\right)$ we are able to witness the impact of the deposition, cancellation and transaction over time. Thus the behaviour seems to be that $v_{1}$ already incorporates time evolution, while $v$ has to be integrated over time. As we shall see, this makes inference from asymptotic analysis easier.

\section{Quantity transport and price auction}

We are intuitively claiming quantity trading. A change in density of market particles on the grid means a reaction if particles with a buy and sell intention occupy a grid position in the LOB (not latent orders). A difference between physical systems and asset markets is that while in the former ensemble measures are defined on the basis of averages, in the latter the representative measure of price is determined by the last transaction, even if it may have involved the minimum allowed quantity. This coupled with low granularity in minimum quantity and non-discretisation of the price grid has led to the possibility of viewing the price auction and quantity auction as separate interconnected mechanisms. We may see auctions resulting in transactions where prices remain stable with low quantity trading or a large amount of quantity traded without price impact or quantity traded with price impact or price impact with low quantity trading. 


\section{Drift and diffusion}

The more market order particles diffuse, new trade transaction prices are formed. But the noise traders can get stuck in longer waiting times in limit orders. The density of the market particles may not allow absorption of volume / quantity of resource density. Sometimes, with targets remaining unconquered the resource particles (limit orders or market orders) have a tendency to move in opposite direction to diffusion of market order particles. We have modelled the impact of this in the drift term that opposes general diffusion. The drift is dependent on the quote revisions from the traders as discussed in example in section 2.2.2.

\section{Bid-ask bounce}

Another interesting facet is the presence of a reaction front characterised by bid-ask bounce. The diffusion through levy search has a tendency to pull the transaction price in opposite direction resulting in the prices moving from the buy side to the sell side. A counter force to this would come from the fact that the participating noise traders would get locked in limit orders and hence the number of transactions they can enter into decreases thereby gradually reducing the efficiency. Sell market orders introduced on the ask side of transaction prices, would need to travel longer to reach the buy side and vice-versa.

We now look into specific scenarios that may operate in the market through simple examples. Such examples can be generated in conjunction with a trading model that employs search as a tatonnement as in the Intraday Trading model of Pani (2019).

\subsubsection{A Steady State Solution}

We want to find a steady state solution to our master equation. Say we have total addition of market particles other than usual as $Q(x, t)$. A steady state solution is an equilibrium solution when the change of market particle density over time is zero. It generally refers to the state where the long term average volume in the asset gets traded. In steady state the diffusion coefficient and drift velocity coefficients are not time dependent. The contribution to market particles coming from $v$ stabilises. In steady state, $v=v_{1}=$ constant i.e the instantaneous change is equal to the total change. At any time $t_{i}$ the net change is effected only at a particular $x$ and not other locations. When price priority rule is used for matching trades, it results in such a general condition in the limit order markets. The rate of particles released from limit orders following transactions is constant and transaction rate is constant. There are two ways to find the solution, first by setting $\frac{\partial g}{\partial t}=0$ or second, we find the solution from the master equation (26) when $t \rightarrow \infty$. 
Our basic equation for the gradient function in the drift velocity expression is (refer Appendix Eq. D.12):

$$
-\left(v(t)-v_{1}(t)\right) \sqrt{\frac{D_{1}(t)}{D(t)}}=\frac{\partial}{\partial t} \sqrt{\frac{D_{1}(t)}{D(t)}}
$$

When $t \rightarrow \infty$. the perceived resource gradient is constant, i.e $\frac{d f}{d x}$ is constant and $\frac{d^{2} f}{d x^{2}}=0$. This implies $v=v_{1}=$ constant. What happens after a long time post addition of $\mathrm{Q}$, with sufficient time to all the three processes to play out to reach an equilibrium state. As $t \rightarrow \infty, v-v_{1}=0$ and $\beta=1$. However, $T$ becomes very large and indeterminate value. The second term of the equation (26) disappears. $T-\zeta=e . \beta_{1}$ is a constant.

Further, since transaction rates are constant and not high $\lambda_{2}=0$. There is no drift, hence $\lambda_{1}=0$. The equation reduces to (27) with the assumption that $\frac{Q(x)}{\sqrt{4 D e}}=\frac{-(x-\chi)^{2}}{4 D e}$. Thus we recover the square root law where $\chi$ is the impact cost. $x-\chi$ is the price region where we will have trades to resolve $Q$.

$$
g(x)=\frac{\beta_{1}}{\sqrt{\pi}} e^{\frac{-Q(x)}{\sqrt{4 D e}}}
$$

\subsubsection{A large demand}

What happens when a large demand $M$ comes into the market. $M$ could be a single large order or a few orders in a short span of time. If the demand is a new market order, it would follow a path trying to move towards a steady state dynamics. Otherwise, after the trades hit this block, it takes a few transactions to lock up the noise particles in the next best limit order position. The market order particles start to sense a gradient and drift begins at a later stage when the locked noise particles are released. The following example illustrates this mechanism that we offer for the market. Fig. 4 to Fig 8 also illustrate some of the discussion below.

\section{Example}

Let $Y_{t}$ denote the transaction price. The grid is denoted relative to the first transaction price $Y_{t 0}$. The continuity to the left and right of $Y_{t 0}$ is the bids and offers respectively, represented by $B B_{1}, B B_{2}, B B_{3}, B B_{4} \ldots$ and $\mathrm{BO}_{1}, \mathrm{BO}_{2}, \mathrm{BO}_{3}, \mathrm{BO}_{4}, \mathrm{BO}_{5} \ldots$ We assume that $Y_{t 0}$ has exhausted the demand and supply at that price. At $t_{0}$ on both sides of $Y_{t 0}$ on the price axis, there will exist a visible order book and a hidden latent order book. Such order books are generally known to take a $V$ or $U$ shape. The bid side demand is at an angle $\theta_{1}$ and the ask side offer is at an angle $\theta_{2}$. This is a visual representation of the $\mathrm{V}$ or $\mathrm{U}$ shape and a easy way to approximate the difference between demand and supply if it exists. The difference between $\theta_{1}$ and $\theta_{2}$ generates a bias in the system, leading to the drift, that we illustrate below. 
Intuitively, market orders want to take the market particles and hence transactions towards $+\infty$ on Ask side and $-\infty$ on bid side. The drift opposes this movement. Let us assume the introduction of $M$, starts with $B O_{1}+B O_{1 h}$, $\mathrm{BO}_{2}$ that is visible and $\mathrm{BO}_{3 h}$ which is hidden. The subscript $h$ is used for the latent / hidden orders. Participant positions are based relative a dynamic value such as the last transaction price (one may alternatively reference either a fundamental value of the asset or an index etc). The introduction of $M$ makes $\theta_{2}>\theta_{1}$, the magnitudes of which is not known. The existence of large demand is revealed only on the basis of visible order book and transactions. At $t=t_{1}$, the transaction price moves to $Y_{t 1} B O_{1}$. The block $M$ now behaves like a sink or wall. It does not allow transactions to move towards $B O_{2}, B O_{3}$. After $Y_{t 1} B O_{1}$, a series of trades can take place at $B O_{1}$ which is the new transaction price $-Y_{t 2} B O_{1}, Y_{t 3} B O_{1} \ldots Y_{t 5} B O_{1}$. Thus, successive transactions $Y_{t 2}, Y_{t 3} \ldots Y_{t 5}$ are recorded at the same price $B O_{1}$. The search for trades is still on in both directions. Once market particles exhaust the liquidity at $B O_{1}$, the trade again reaches $Y_{t 0}$ which is the new best bid. New transaction price is $Y_{t 6} Y_{t 0}$. If no liquidity is available here, the levy search can move to either $B O_{1}$ or $B B_{1}$. Since, the former keeps locking up the noise traders in limit orders, more likely the trade moves to $B B_{1}$ within a couple of cycles. Hidden liquidity can reappear at $Y_{t 0}$ only if there is a readjustment of positions. By this time, the market particles system has made sense of the gradient and the drift can get activated. This simply involves correcting the bias $\theta_{2}>\theta_{1}$.

The correction of bias is the action based on readjustment of position on either side. The reference point $Y_{t 0}$ needs to move to $B O_{1}, B O_{2}$ or $B B_{1}, B B_{2}$. This is the drift in action and usually takes place when participants revise their position on the basis of new information or are not satisfied with the slow rate of trades. Once transaction pace picks up on either side, there could be a new source of drift from released market particles. These particles can get involved in the carry trade for a small margin. In the absence of new information and no adjustment on bid side, the transaction price gradually moves up from $B B_{1}, B B_{2}$. The best offers start to move from $B O_{1}$ to $Y_{t 0}$, $B B_{1}$. In this situation, the demand $M$ can face the lack of liquidity forcing a readjustment as illustrated in fig 6-8.

When $M>>G_{i} \omega(\chi)$ we can ignore the second term in the equation. The expression for density reduces to:

$$
g(x, t)=e^{v^{*} t} \frac{M}{\sqrt{4 \pi D T}} \exp \left[\frac{-1}{4 D T} e^{-v^{*} t}\left(x-\int_{0}^{t}\left(\lambda_{2}+\lambda_{1} \phi_{2}\right) d s\right)^{2}\right]
$$

In (28), what happens if market orders are time dependent and not simply as a response to one very large order. We assume this large input of market orders is large enough to assume the initial density to be very low So, $Q(x, t)=G_{0} f(0, t) \delta(x)$. Here the function $f(0, t)$ shows the continuous availability of market orders at a price. This could be in response to liquidity demand in the order book in limit orders or the perceived hidden limit orders. This liquidity is the source of large liquidity. Further from the equation below for gradient observed by 


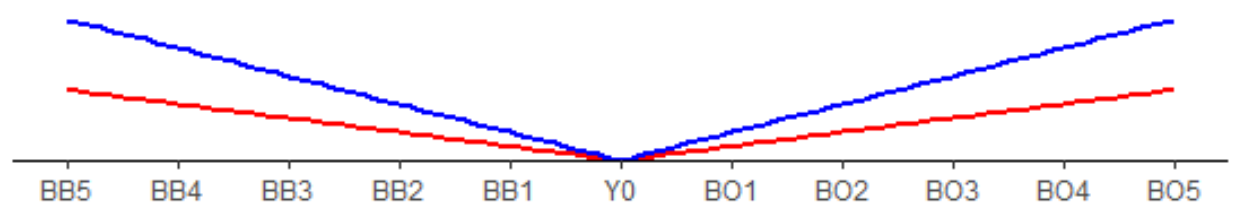

Figure 4: Visual representation of a balanced total order book, visible orders shown in red and the cumulative visible and hidden orders in blue. Hidden orders comprise the orders that may be placed to demand or supply liquidity. The hidden limit orders are placed at a later time and the hidden market orders are placed at the particular transaction prices. The y-axis is the quantity. $Y 0$ is the last transaction price. The bid side demand is at an angle $\theta_{1}$ and the ask side offer is at an angle $\theta_{2}$. Here, $\theta_{1}=\theta_{2}$. Fig 4 to Fig 8 is a sequential illustration showing biases due to new supply demand addition and resulting transactions and adjustments due to quote revision.

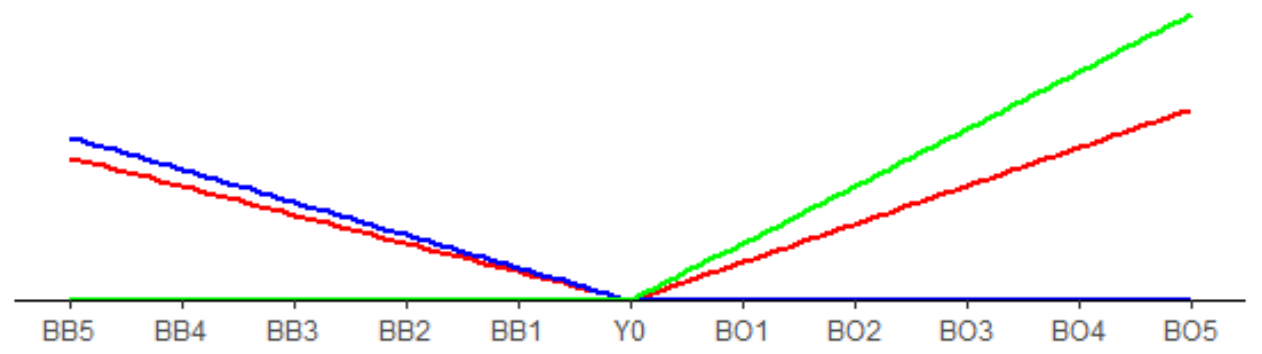

Figure 5: A bias develops around the last transaction price given in fig 4. Visible orders shown in red and cumulative with hidden demand in blue and cumulative with hidden supply in green. The y-axis is the quantity. $Y 0$ is the last transaction price.A large demand $M$ is added at $\mathrm{BO} 1$ (visible+hidden), $\mathrm{BO} 2$ (visible+hidden), $\mathrm{B} 03$ (hidden). The bid side demand is at an angle $\theta_{1}$ and the ask side offer is at an angle $\theta_{2}$. Here, $\theta_{2}>\theta_{1}$ a demand supply mismatch that leads to bias in the system due to hidden orders. (Fig 4 to Fig 8 is a sequential illustration showing biases due to new supply demand addition and resulting transactions and adjustments due to quote revision) 


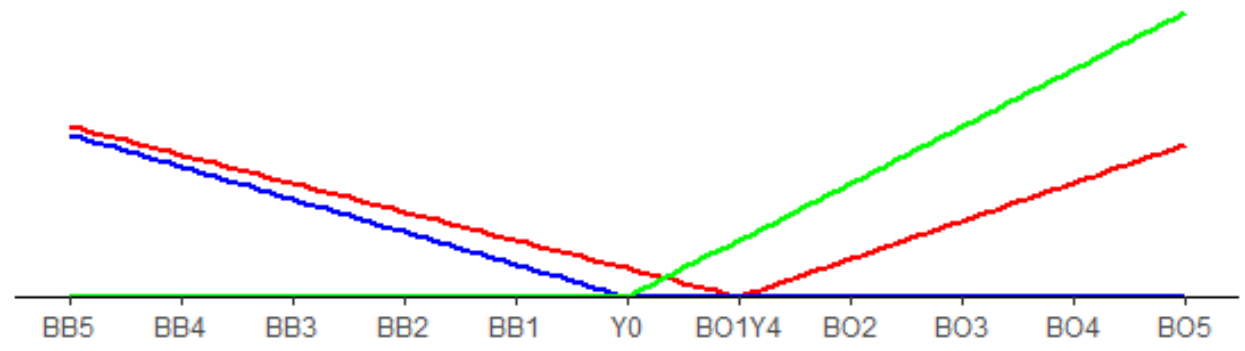

Figure 6: After a few transactions from fig 5, the visible order book moves to new last transaction price $B O 1 Y 4$ as reference by adjustment of limit orders ( $Y 4$ is the fourth tansaction price). Visible orders shown in red and latent demand in blue and latent supply in green. The latent order book is referenced to a fundamental price or the equilibrium price from the initial auction. $Y 0$ is the first transaction price in the illustration. The y-axis is the quantity. (Fig 4 to Fig 8 is a sequential illustration showing biases due to new supply demand addition and resulting transactions and adjustments due to quote revision)

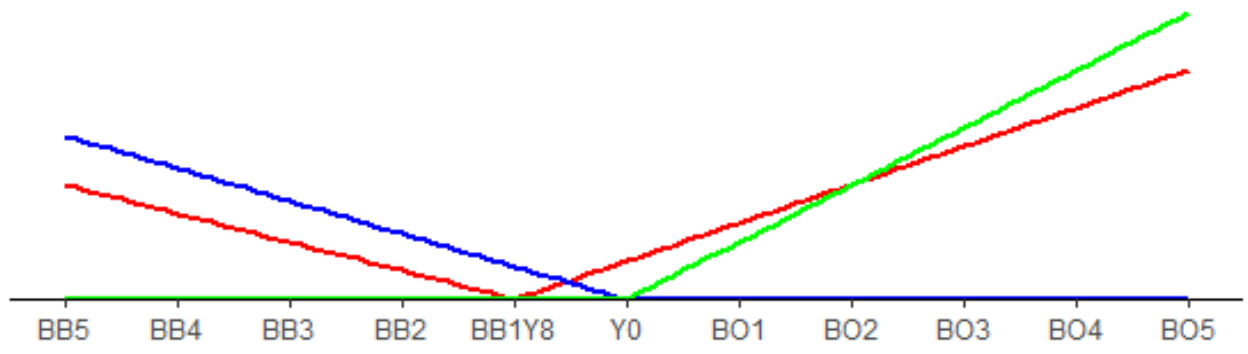

Figure 7: A few transactions afterfig 6, the visible order book moves to new transaction price $B B 1 Y 8$ as reference by adjustment of limit orders. The movement is from BO1 to BB1. Visible orders shown in red and hidden demand in blue and hidden supply in green. Note the bias adjusting in visible orders but the bias in hidden order remains with $\theta_{1}$ and $\theta_{2}$, the relative angles with the axis remaining the same Fig 4 to Fig 8 is a sequential illustration showing biases due to new supply demand addition and resulting transactions and adjustments due to quote revision)

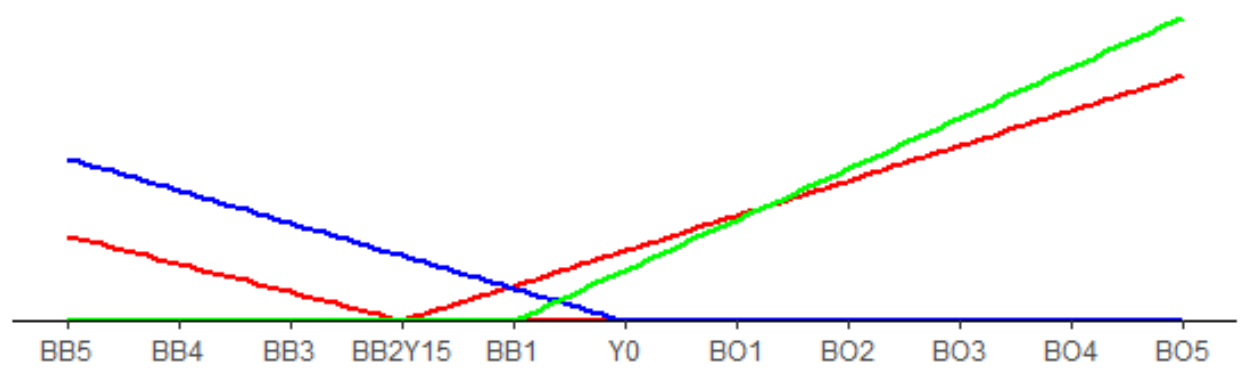

Figure 8: The transaction price (Y15) moves further to BB2Y15 by the levy search. Visible orders shown in red and hidden demand in blue and hidden supply in gree ${ }_{4}$ The bias due to supply corrects as it dissociates from demand through quote revision and moves the reference point to BB1 reducing $\theta_{2}$. (Fig 4 to Fig 8 is a sequential illustration showing biases due to new supply demand addition and resulting transactions and adjustments due to quote revision) 
liquidity providers (refer Appendix D.9):

$$
f(x, t)=\frac{v(t)-v_{1}(t)}{\lambda_{1}(t)} x^{2}+\phi_{2}(t) x+c
$$

We infer that $Q=\phi_{2}(t)$. In such situations $\lambda_{2}$ can be ignored and $v_{1}, v-v_{1}$ can be reduced to $v *$ to further simplify the equation.

$$
\begin{gathered}
g(x, t)= \\
\beta_{1} \int_{0}^{t} \frac{\phi_{2}}{\sqrt{4 \pi D(T-\zeta)}} \exp \left[\frac{-1}{4 D(T-\zeta)} e^{-v^{*} t}\left(\frac{x}{\beta(t)}-\int_{0}^{t}\left(\frac{\lambda_{2}}{\beta(t)}+\frac{\lambda_{1} \phi_{2}}{\beta(t)}\right) d s\right)^{2}\right]
\end{gathered}
$$

\subsubsection{High reaction rate}

When the reaction rate is high, we evaluate the dynamics under the further assumption of no addition of market orders through $q(x, t)$. We expect the density $g$ at the transaction price to go down. This is represented as (30), which is the second term in (26).

$$
\begin{gathered}
g(x, t)= \\
\beta_{1}(t) \int_{-\infty}^{\infty} \frac{1}{\sqrt{4 \pi D T}} G_{i} \omega(\chi) \exp \left(-\frac{1}{4 D T}\left(\frac{x}{\beta}-\int_{0}^{t}\left(\frac{\lambda_{2}}{\beta}+\frac{\lambda_{1} \phi_{2}}{\beta}\right) d s-\chi\right)^{2}\right) d \chi
\end{gathered}
$$

Such a situation could arise if the market orders hit a liquidity pool in limit orders and a large number of noise traders that were earlier locked up in limit orders here get released. The liquidity pool causing high transaction rates and release of noise traders creates a sustainable transaction rate. The contribution to $v$ is coming only from the reaction rate. $\phi_{2}=0$ and (30) reduces to (31).

$$
g(x, t)=\beta_{1}(t) \int_{-\infty}^{\infty} \frac{1}{\sqrt{4 \pi D T}} \omega(\chi) \exp \left(-\frac{1}{4 D T}\left(\frac{x}{\beta}-\int_{0}^{t}\left(\frac{\lambda_{2}}{\beta}\right) d s-\chi\right)^{2}\right) d \chi
$$

\subsubsection{Drift has no time dependence and low reaction rate}

In case, the drift is not time dependent and only spatially dependent and no self generated drift resulting from release of noise traders. $\lambda_{2}=0$ and the drift in response to resource gradient reduces to (32)

$$
\lambda_{1}(t) \frac{\partial f(x, t)}{\partial x}=\lambda_{1} \frac{\partial f(x, t)}{\partial x}=\lambda_{1}\left(\frac{v-v_{1}}{\lambda_{1}} x\right)+\lambda_{1} \phi_{2}(t)
$$




\subsubsection{Continuous spatial inflow of market orders that is not time dependent}

This is a case of liquidity available at every price in hidden market orders. In such a case we need to find the equivalent of (29) that is spatially dependent and not time dependent,

$$
g(x, t)=\beta_{1}(t) \int_{0}^{t} \int_{-\infty}^{\infty} \frac{\left(v-v_{1}\right) \chi}{\sqrt{4 \pi D(T-\zeta)}} \exp \left(\frac{-1}{4 D(T-\zeta)}\left(\frac{x}{\beta}-\chi\right)^{2}\right) d \chi d \tau
$$

If dispersion $D$ too is assumed to be time independent $D=D_{0}$ and we assume $v, v-v_{1}=v *$ because this is a small value, (33) reduces to (34). ( $D$ could take other forms too).

$$
g(x, t)=e^{v^{*} t} \int_{0}^{t} \int_{-\infty}^{\infty} \frac{v^{*} \chi}{\sqrt{4 \pi D_{0}(T-\zeta)}} \exp \left(\frac{-1}{4 D_{0}(T-\zeta)}\left(e^{v^{*} t} x-\chi\right)^{2}\right) d \chi d \tau
$$

\subsubsection{At the limit of continuous auctions}

What happens as $t \rightarrow 0$ immediately after a transaction / auction has ended. Once a transaction price is established new market orders come in at that price. These market orders could be buy or sell orders or both. Other than such deposition we assume cancellation and transaction do not occur at this instance. Since, in this scenario $v=v_{1}=$ constant,$\beta=1$ and $\beta_{1}=1$. However, $T$ and $(T-\zeta)$ does not exist and we cannot determine the density of market order particles. We did not evaluate this limit for the levy search. However, in our model of levy search process we know that market orders start coming in at the last transaction price with a velocity drawn from a distribution and that the process exists. This will provide the result to the time derivative at that point. It may be meaningful to find what happens in the inter-trade interval between two trades. The average of this interval is precisely the average trade duration. Let two consecutive trade transactions take place at time $t_{1}$ and $t_{2}$, $\left|t_{2}-t_{1}\right|=\tau$. What happens in this regime will depend on the dominant process occurring in the market: diffusion / levy search, drift due to high transaction rate, drift due to perceived resource gradient. We keep mixed processes out of the scope of the current analysis.

In the diffusive regime, the contributions from other processes is neglected. Further, further we assume $Q=0$ as presence of large market orders will invoke other processes, $\beta=\beta_{1}=e^{v^{*} \tau}, T$ reduces to $\left(2 v^{*} e^{2 v^{*} \tau}\right)^{-1}$. In (35) $B$ is the bid-ask spread. (35) reduces to (36) on simplifying. (36) gives the relationship between the initial density of particles, diffusion coefficient, spread and interauction time with the density of particles. To understand the intuition in (36) we further assume that the liquidity demand at $Y_{t_{2}}$ comes from limit orders and the market particles provide liquidity. To find the maximum capacity during the inter-auction trade (we assume availability of targets is not a constraint), let us assume the ideal condition that every particle that engages in search reaches the 
target. And that every new addition even in the period $\tau$ is only a deposition that can go in and provide liquidity. So both $D$ and $v^{*}$ are equal to 1 . Fig. 9 and Fig. 10 gives multiplying factor to the intial density as a function of the spread and interauction times.

$$
\begin{gathered}
g\left(Y_{t_{2}}\right)=e^{v^{*} \tau} \frac{\omega\left(Y_{t_{2}}\right)}{\sqrt{4 \pi D\left(2 v^{*} e^{2 v^{*} \tau}\right)^{-1}}} \exp \left(\frac{-1}{4 D\left(2 v^{*} e^{2 v^{*} \tau}\right)^{-1}}\left(\frac{B}{e^{v^{*} \tau}}\right)^{2}\right) \\
g\left(Y_{t_{2}}\right)=\frac{\omega\left(Y_{t_{2}}\right)}{\sqrt{4 \pi D\left(2 v^{*}\right)^{-1}}} \exp \left(2 v^{*}\left(\tau-\frac{B^{2}}{4 D}\right)\right)
\end{gathered}
$$

Fig. 9 confirms that in an ideal best search scenario the density exists. As the spread increases, the density is reduced. At spreads of 0.2 and below the density converges to the initial density of particles in interauction times of 0.5 seconds. Fig. 10 magnifys the fig. 9 around the low interauction times prevalent in liquid equity markets. For the spreads of 0.2 and below it shows convergence around the constant $(1 / \sqrt{2 \pi})$ in (36). This suggest the exponent of $e$ is 0 that is the expression $\left(\tau-\frac{B^{2}}{4 D}\right) \approx 0$. Thus we expect in a fairly ideal scenario 40 percent of the initial density of market particles is able to trade. This demonstrates how the presence of arbitrageurs and other traders, who contribute to the market particles, can help increase the depth of the market. Note that this is the range we would obtain every time the efficiency of the search is equal to the contribution from the addition.

The relationship between the spread and diffusion coefficient can be understood from the need for the following to hold: $B<2 \sqrt{D}$ so that the exponent is positive. The diffusion coefficient could be calculated in the standard way as MSD upon average flight time.

In the regime of drift due to high transaction rates, we ignore drift due to any resource gradient. The scenario is that a large number of market particles are already released due to high transaction rates before we arrive at $t_{1}$. Since this component is significantly higher than any new orders, $v_{1}>>v$, hence, $\beta=e^{-v^{* \tau}}$ and $\beta_{1}=e^{v^{* \tau}}$ and $T=\frac{e^{2 v^{* \tau}}}{2 v^{*}}$. In place of (36) we arrive at (37), where we can see that if the spread is high the contribution from this drift will be small towards making market particles available for transaction.

$$
g\left(Y_{t_{2}}\right)=\frac{\omega\left(Y_{t_{2}}\right)}{\sqrt{4 \pi D\left(\frac{1}{2 v^{*}}\right)}} \exp \left(-\frac{2 v^{*}}{4 D}\left(B-\lambda_{2}^{*} \tau\right)^{2}\right)
$$

When under the process of drift due to resource gradient, $v>>v_{1}$ and we neglect $v_{1}$. The equivalent expression is given in (38). Again a high spread can reduce the contribution of this process in the limit under consideration.

$$
g\left(Y_{t_{2}}\right)=\frac{\omega\left(Y_{t_{2}}\right)}{\sqrt{4 \pi D\left(\frac{1}{2 v^{*} e^{2 v^{*} \tau}}\right)}} \exp \left(-\frac{1}{4 D\left(\frac{1}{2 v^{*}}\right)}\left(B-\lambda_{1}^{*} \phi_{2}^{*} \tau\right)^{2}\right)
$$




\section{Density in interauction time in Best Search scenario}

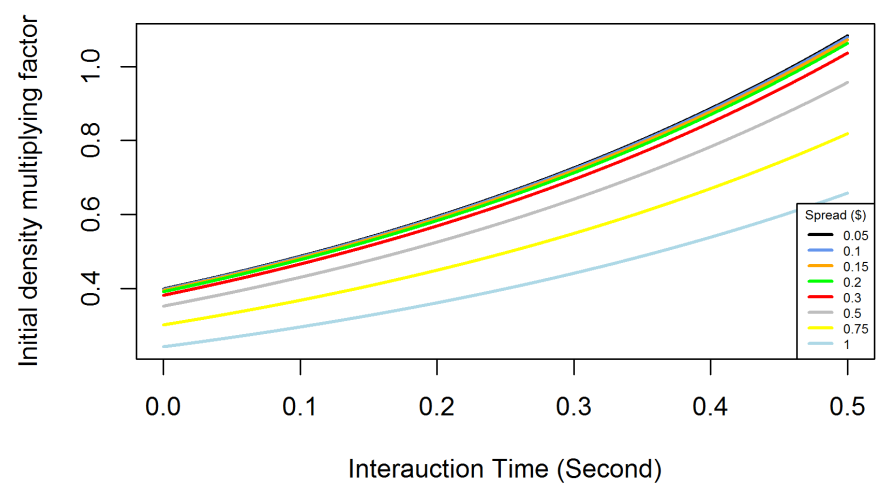

Figure 9: The multiplying factor to the initial density as a function of the interauction time $(\tau)$ and spread $(B)$. The multiplying factor gives the proportion of initial density of particles that are traded.

\section{Density in interauction time in Best Search scenario}

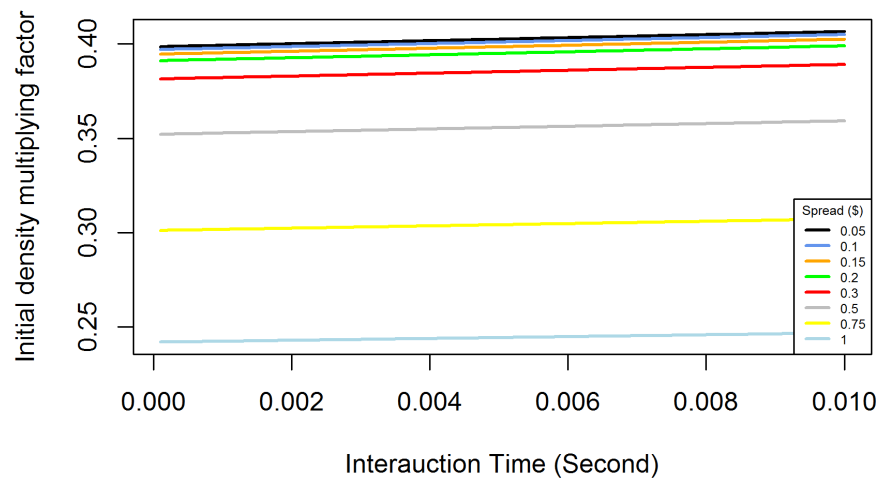

Figure 10: The multiplying factor to the initial density as a function of the interauction time $(\tau)$ and spread $(B)$. This figure magnifys the fig 6 (a) around the low interauction times prevalent in liquid equity markets. 


\section{NUMERICAL SIMULATION}

We performed a numerical simulation of the model to improve our understanding of the results. The numerical simulation is different than the regular reaction-diffusion simulation because while orders may be present in the total order book, they come into the limit order book at different times. Researchers have in the past modelled the arrival of orders into the limit order book using a poisson or a hawkes process (Gould et al. (2013)). Further, limit orders may get cancelled and leave the order book or may get cancelled and get modified, the latter is usually treated as a new order.

\subsection{Pseudo-experiment set up}

We begin with a market where the number of traders in the market is fixed at 1000. A trader places only a single order. An order is equivalent of a particle discussed in section 2.

\subsubsection{Simulating Bias}

There are in all two sets of simulation that differ only in one aspect: in the first set, the quantity of shares in the trader's order is one while in the second set, it may vary between one to five. We use this difference in ordered quantity of shares to simulate the bias. The minimum order quantity is one share.

\subsubsection{Simulating Resource Density: order book events}

The arrival of events (order arrival, order cancellation, transactions) is modelled following a poisson process. While transactions are not orders, this seems to be a practical alternative to represent the transactions in the tick by tick data as and when they occur. Thus, an event of order arrival / order cancellation is followed by a order matching to determine the possibility of transaction. If there is a transaction, it is posted with a timestamp of the subsequent event arrival time, else the next order arrives at the timestamp. The event arrival rate is one of the determinants of the resource density.

\subsubsection{Traders}

The percentage of noise traders in the market is assigned randomly in each experiment drawn from a uniform distribution. The quoting and cancellation behaviour is governed by the velocity and flight time distributions. The simulation assumes a cauchy velocity distribution (ref Eq. 6) and a flight time distribution with power tails (ref Eq. 7). The simulation is initialised with a starting price or known price for the asset and the traders assigned a velocity and a flight time, both drawn from the distribution. Traders are randomly assigned as a noise or strategic 
trader. This is subject to total number of noise traders in the experiment. The sign of the velocity distinguishes the buyers and sellers.

Type of order choice - Limit or market is randomly assigned. The product of velocity and flight time provides the deviation of price quote decision by the trader from the initialised starting price. This is relevant for the strategic traders who can start by placing limit orders, as noise traders always start with market orders. Strategic traders exit the market after the transaction or the orders are cancelled at the end of the flight time. Noise traders after the initial placement, alternate between market orders and limit order placement and exist in the market till they are able to buy the asset with available funds or till end of flight time. The pricing rule for limit order placement by Noise traders is with a spread of 0.1 from the last transaction price.

\subsubsection{Event arrival rates and experiments}

Event arrival rates vary for thickly versus thinly traded stocks. For each stock event arrival rates can vary within the trading day. The set of event arrival rates (measured as events per second) included in the experiment is $\{1,6,10,100,1000,10000\}$. The included set covers a wide spectrum of event arrival rates and hence the resource density. It covers the necessary range, but is not dynamic i.e does not vary through the trading period. The included set for $\gamma$ (power of flight time distribution from (7)) is $\{0.25,0.5,0.75,1,1.25,1.5,1.75,2,2.25,2.5,2.75\} . \gamma>2$ is the region of Brownian search, $1<\gamma<2$ is the region of superdiffusive or levy search, $\gamma<1$ is the region of ballistic search. 30 experiments each are simulated for each pair of event arrival rate and $\gamma$. This design results in a total of 3960 experiments (two sets of 1980 experiments each for bias and non-bias case)

\subsection{Results}

Fig. 11 gives the results of the numerical simulation (no-bias case) showing the rate of trades (identified as the search efficiency) per event arrival rate. Since the quantity quoted and traded is one unit, there are no biases arising due to resource density in this set of simulation. The simulation could be further extended by including a trading model and responses of traders to a bias. Such a simulation could demonstrate the search efficiency of examples discussed in section 2.2.1-2.2.6.

Unlike ballistic search, search efficiency is dependent on the event arrival rate (resource or target density) in both brownian and superdiffusive search. This is in line with the findings in Palyulina et al. (2014). When trading intensity is low (event arrival at 1 to 100 per second), super-diffusive and ballistic search is more efficient than brownian search with ballistic search being the most efficient. When the resource density is high with event arrivals at 1000 events per second or higher, both diffusive and superdiffusive search is equally efficient. In the 


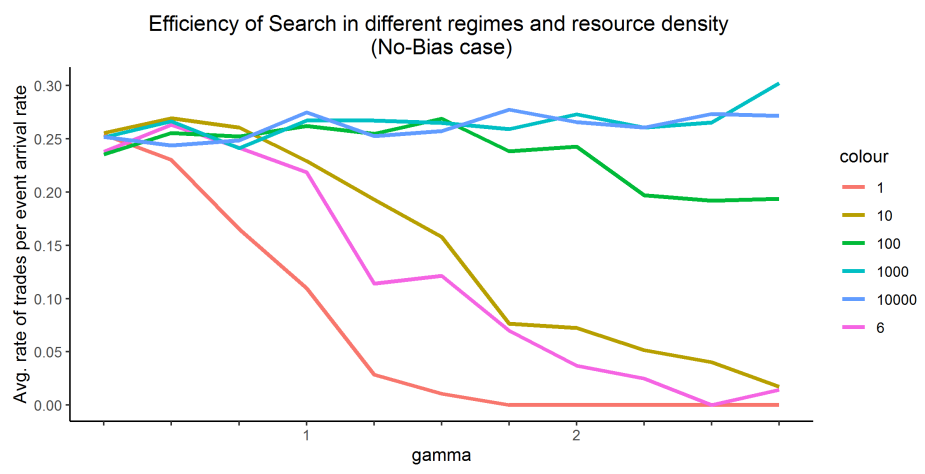

Figure 11: The efficiency of search depends on the search regime and the resource density. The former is set through the quoting and cancellation behaviour. And the latter through the event arrival rates. In low resource density, superdiffusive $(1<\gamma<2)$ and ballistic search $(\gamma<1)$ perform better. When resource density increases and in absence of any biases in the environment, brownian search is as efficient as the others.

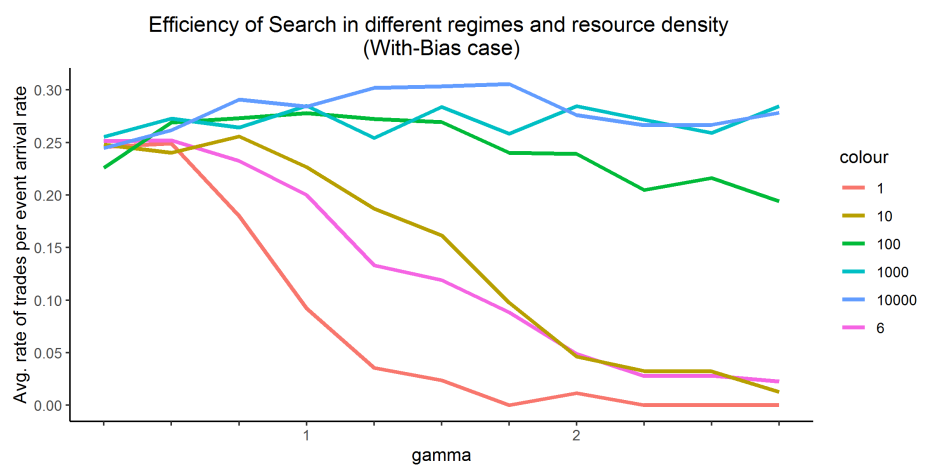

Figure 12: The efficiency of search depends on the search regime and the resource density. The former is set through the quoting and cancellation behaviour. And the latter through the event arrival rates. Additionally, the presence of Bias due to resource density increases efficiency of superdiffusive search more than brownian search. In low resource density, superdiffusive $(1<\gamma<2)$ and ballistic search $(\gamma<1)$ perform better. 

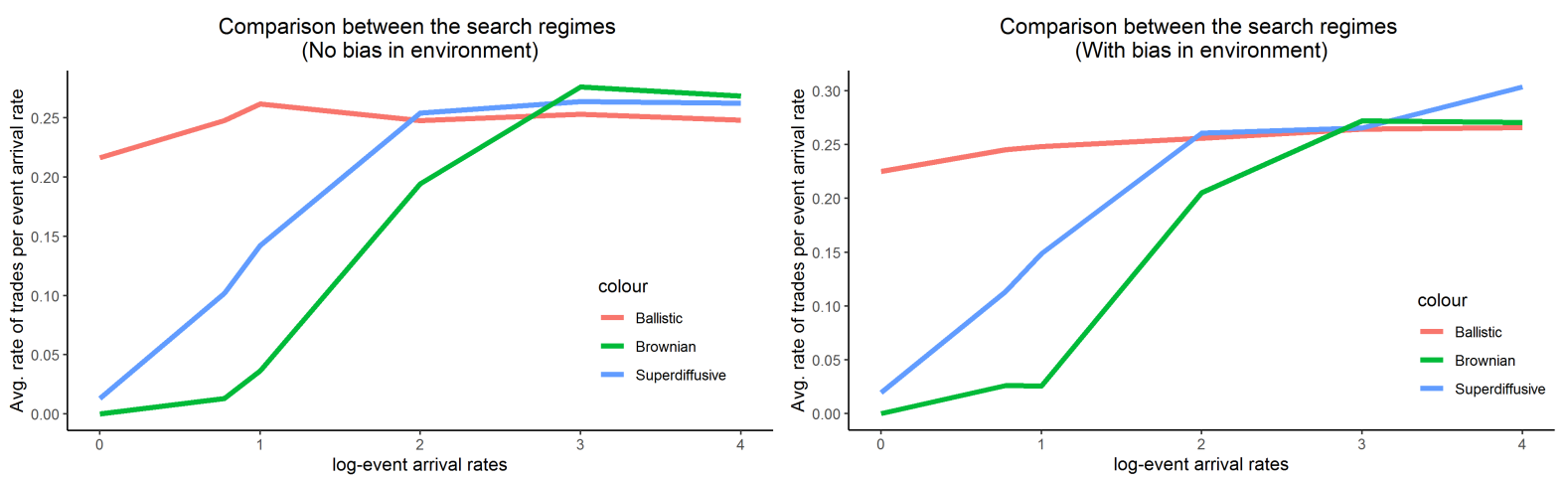

Figure 13: Comparison of the search regimes in different resource density and influence of bias. In the presence of bias, superdiffusive search is more efficient than brownian. Ballistic search performs the best in low resource density and is less influenced by resource density

presence of bias (fig. 12) however, the superdiffusive search begins to perform better even when the resource density is very high (event arrival rates of 10000 per second).

Fig. 13 gives a comparison of the average trading rates under the three search regimes normalised to event arrival rates. Superdiffusive search is more efficient than Brownian in the presence of bias. Brownian search performs better in the absence of bias and in an environment of high resource density. These are clearly related to active trading periods of liquid stocks. We do not find the rate of trades being impacted by the relative presence of noise traders (fig. 14). The poisson arrival of events (traders and orders) into the market could be the possible reason.

Fig. 15 gives a sample of the trade prices obtained under different event arrival rates, for the case when $\gamma=1.5$ and traders can quote different quantities (bias in environment). The price time plots describe the behaviour of prices. The saw-tooth property of transaction price equilibriation in double auctions markets (Plott $(2008))$ can be observed (except with event arrivals at rate of 10000/sec).

\section{DISCUSSION AND CONCLUSION}

We have introduced and analysed a model, to show how auctions in high frequency markets without a designated market maker can be described as a search by buyers for a seller and vice-versa. The model is based on a zero intelligence approach. We consider a Total order book model that includes a limit order book and the latent order book.

Intuitively, when the principle for matching trades is based on 'price-time priority' in a continuous double auction mechanism, it seems to resemble a search. The need for the model arises as existing theory based on 


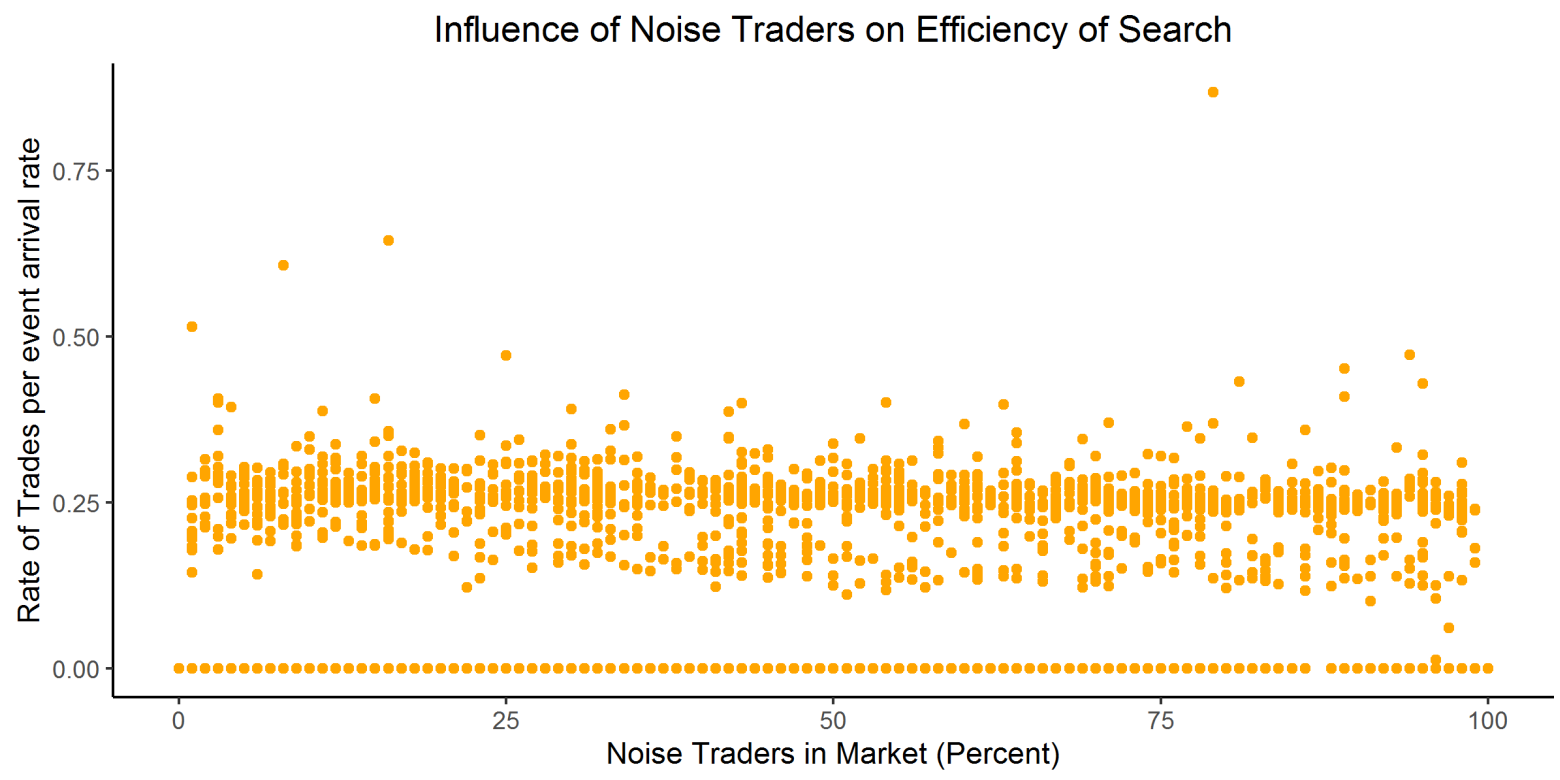

Figure 14: The percentage of noise traders in each trial of the simulation is randomly assigned. The number of noise traders does not affect the efficiency of search. The possible reason could be the poisson arrival of the traders. The above is the no-bias case that plots the rate of trades per event arrival rate with the percentage of noise traders in the market. The plot of the with-bias case is also similar.

supply and demand assumes the existence or emergence of a walrasian equilibrium. The above assumption takes the underlying model away from reality when considering intraday markets that trade assets in high frequency through continuous auctions. In high resolution the assumption of an equilibrium of demand and supply leading to optimised quantity traded and emergence of price is fictional. This is especially true in case of order driven markets without designated market makers. The general belief that in financial markets given that trading is continuous, prices can quickly adjust to clear the market is valid but the process is not instantaneous. Information flow is not seamless and frictions exist. Traders are not always present in the market and alternate opportunities exist for the traders. Market makers and dealers in quote driven markets were the key to push the trading to equilibrium.

Our model is a diffusion-drift-reaction model and inspired by search in biology and robotics. We analyse a number of asymptotic relationships in the model. In the limit of continuous auctions we are unable to determine the density of market particles or trading activity (similar to Donier and Bouchaud (2016)). We analyse the interauction times in which the density exists and a relationship between the diffusion coefficient, interauction time, initial density of particles and spread exists. For the spread less than 0.2 USD and inter-auction time, 0.001 seconds or lower, less than 40 percent of the existing market particles are able to trade. There exists a relationship between the spread and the diffusion coefficient for an efficient market. So markets that also depend on drift 

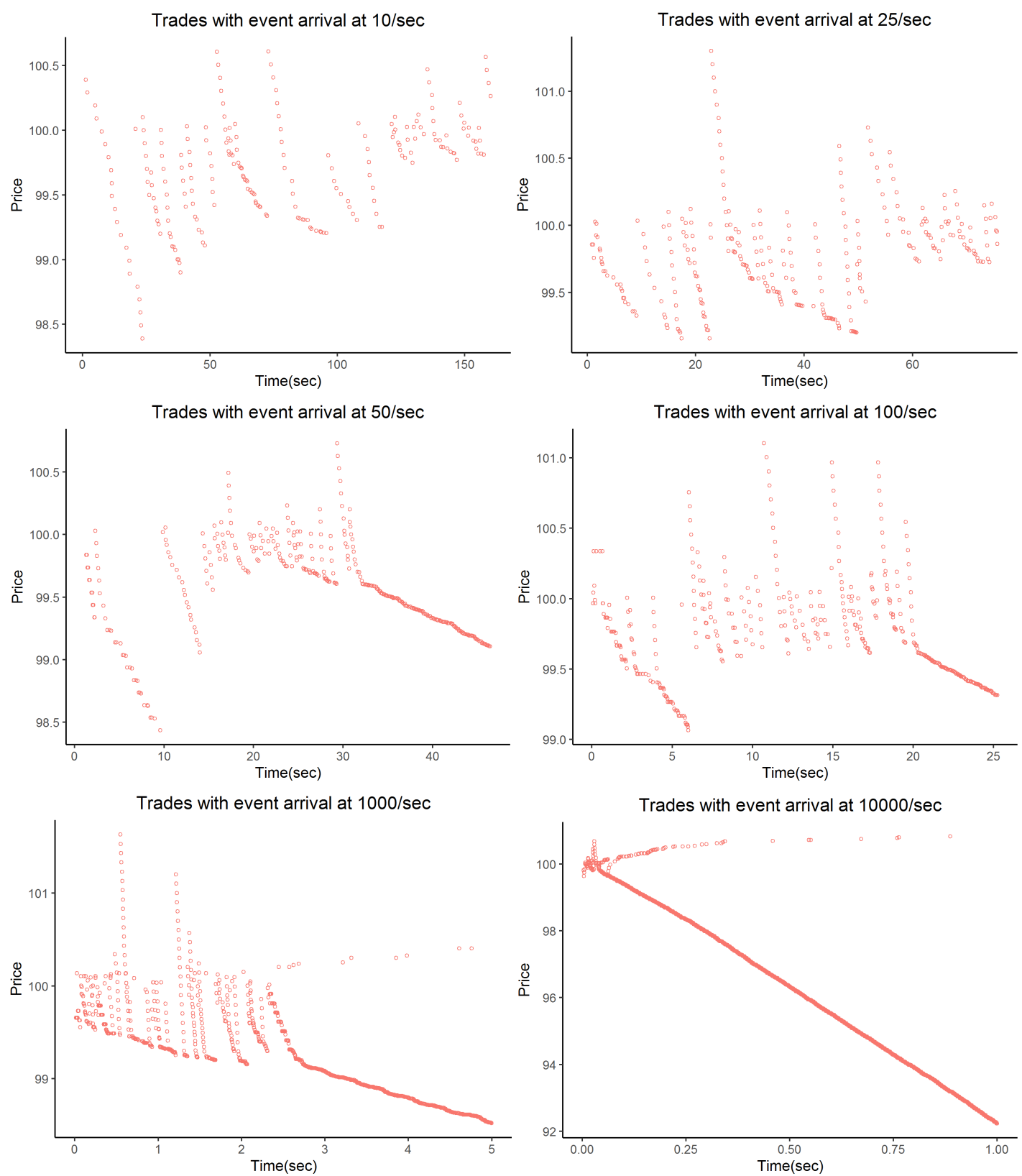

Figure 15: The behaviour of prices under the model. The plots of price and time provides insight into the behaviour of prices. All samples are for trade prices were obtained in the numerical simulation under an environment of bias, i.e traders can quote different quantities. The parameter $\gamma=1.50$, signifying superdiffusive search regime. The plots represent various rates of event arrival - 10, 25, 50,100,1000, 10000 per second. The event arrivals includes order arrivals and a driver of the resource density. 
(resulting from high transaction rates involving noise traders) apart from diffusion are adversely impacted when the spread is high.

The numerical simulation run on the model brings out the search efficiency of different search regimes (ballistic, superdiffusive and brownian) in the simulated set up. The rate of trades that emerges from trade duration is a natural candidate to measure the efficiency of search. When biases exist due to resource density, the superdiffusive search performs better than brownian search. In the absence of bias brownian search is equally efficient in high resource density. In low resource density ballistic search performs the best followed by superdiffusive search. Performance of ballistic search is relatively not affected by resource density or the presence of bias.

Future research can attempt to connect different elements of market design, such as spread, tick size, presence of market maker etc, to the search regimes to understand appropriate design basis the resource density.

\section{Acknowledgement}

We gratefully acknowledge valuable comments received, from an anonymous referee on an earlier version of this paper and two anonymous referees on the current version, that has improved this manuscript.

\section{References}

P. Bak, M. Paczuski, and M. Shubik. Price variations in a stock market with many agents. Physica A: Statistical Mechanics and its Applications, 246(3-4):430-453, December 1997.

Shmuel Baruch and Lawrence R Glosten. Tail expectation and imperfect competition in limit order book markets. Journal of Economic Theory, 183:661-697, 2019.

Avraham Beja and M Barry Goldman. On the dynamic behavior of prices in disequilibrium. The Journal of Finance, 35(2):235-248, 1980.

Avraham Beja and Nils H Hakansson. Dynamic market processes and the rewards to up-to-date information. The Journal of Finance, 32(2):291-304, 1977.

Michael Benzaquen and Jean-Philippe Bouchaud. A fractional reaction-diffusion description of supply and demand. The European Physical Journal B, 91(2):23, 2018. 
Jonathan Brogaard, Terrence Hendershott, and Ryan Riordan. Price discovery without trading: Evidence from limit orders. The Journal of Finance, 74(4):1621-1658, 2019.

S. Denisov, V. Zaburdaev, and P. Hanggi. Levy walks with velocity fluctuations. Physical Review E, 85(3), March 2012.

J Donier and J-P Bouchaud. From Walras auctioneer to continuous time double auctions: a general dynamic theory of supply and demand. Journal of Statistical Mechanics: Theory and Experiment, 2016(12):123406, December 2016.

J. Donier, J. Bonart, I. Mastromatteo, and J.-P. Bouchaud. A fully consistent, minimal model for non-linear market impact. Quantitative Finance, 15(7):1109-1121, July 2015.

D. Froemberg, M. Schmiedeberg, E. Barkai, and V. Zaburdaev. Asymptotic densities of ballistic Levy walks. Physical Review E, 91(2), February 2015.

C Godreche and JM Luck. Statistics of the occupation time of renewal processes. Journal of Statistical Physics, 104(3-4):489-524, 2001.

Martin D. Gould, Mason A. Porter, Stacy Williams, Mark McDonald, Daniel J. Fenn, and Sam D. Howison. Limit order books. Quantitative Finance, 13(11):1709-1742, November 2013.

Daniel Grunbaum. Using Spatially Explicit Models to Characterize Foraging Performance in Heterogeneous Landscapes. The American Naturalist, 151(2):97-113, February 1998.

Richard Haberman. Elementary applied partial differential equations: with Fourier series and boundary value problems. Prentice-Hall, Englewood Ciffs, N.J, 2nd ed edition, 1987.

Joel Hasbrouck. Price Discovery in High Resolution*. Journal of Financial Econometrics, 09 2019. ISSN 1479-8409. doi: 10.1093/jjfinec/nbz027.

Paul Jusselin, Thibaut Mastrolia, and Mathieu Rosenbaum. Optimal auction duration: A price formation viewpoint. Available at SSRN 3399151, 2019.

Albert S Kyle, Anna A Obizhaeva, and Yajun Wang. Smooth trading with overconfidence and market power. The Review of Economic Studies, 85(1):611-662, 2018.

Maureen O’Hara. High frequency market microstructure. Journal of Financial Economics, 116(2):257-270, 2015. 
Vladimir V Palyulina, Aleksei V Chechkinb, and Ralf Metzlera. Levy flights do not always optimize random blind search for sparse targets. PNAS, 111(8):2931-2936, 2014.

Sudhanshu Pani. The information content of quotes in high frequency order driven markets. Unpublished Manuscript, 2019.

Charles R Plott. Properties of disequilibrium adjustment in double auction markets. Handbook of experimental economics results, 1:16-21, 2008.

E P Raposo, S V Buldyrev, M G E da Luz, G M Viswanathan, and H E Stanley. Levy flights and random searches. Journal of Physics A: Mathematical and Theoretical, 42(43):434003, October 2009.

Andy M. Reynolds. Current status and future directions of Levy walk research. Biology Open, 7(1), January 2018.

Abhishek Sanskrityayn and Naveen Kumar. Analytical solution of advection diffusion equation in heterogeneous infinite medium using Greens function method. Journal of Earth System Science, 125(8):1713-1723, December 2016.

V. Zaburdaev, S. Denisov, and J. Klafter. Levy walks. Reviews of Modern Physics, 87(2):483-530, June 2015.

Vasily Zaburdaev, Michael Schmiedeberg, and Holger Stark. Random walks with random velocities. Physical Review E, 78(1), July 2008.

\section{Appendix}

\section{A The Search for trades in absence of bias}

Let $u(x, t)$ be the probability density function (pdf) of the distance travelled by market order particles in search of trades. Thus, $x$ is the total length of the jumps and $t$ is the total time. The velocity $v$ imparted by traders on particles can have positive and negative values to include buy or sell orders (direction of motion). If ' $N$ ' number of trades takes place in a unit of time $d t$, the average duration can be given by $\langle\mu\rangle=d t / N$. Each inter-trade period is a search for the next transaction. A number of jumps get recorded in this period. Equation (39) gives the total path traversed in the search till time $t$. The initial distribution of the market particles is given by $n_{0}(x)$.

$$
u(x, t)=\int_{-\infty}^{\infty} d v \int_{0}^{t} u(x-v \tau, t-\tau) h(v) f(\tau) d \tau+n_{0}(x) \delta(t)
$$


The pdf of the traded particles, $q(x, t)$ is the number of trades that take place in a time interval $(0, t)$. However, the trades being the success of the search of market particles in a tatonnement, we define the efficiency $\eta$ of the search process as the ratio of the total path traversed in the search to the number of targets searched or trades done. This gives us a link to represent $q(x, t)$ in terms of $u(x, t)$ and $\eta$ as in equation (40). $\eta$ is again a function of the coupling of velocity imparted and the flight times.

$$
q(x, t)=\int_{-\infty}^{\infty} d v \int_{0}^{t} \frac{u(x-v \tau, t-\tau) h(v) f(\tau)}{\eta(v \tau)} d \tau
$$

Equations (40) and (41) fully describe the dynamics of the system with a given initial density of particles and the two pdf for the flight times and velocities. They establish the crucial link between the tatonnement in the auction with the trades. Next we solve these equations analytically. First, we determine the total path traversed by market particles in the search process and then introduce the result into the equation of the trade density. We apply the Fourier transform with respect to the spatial coordinate in (39). Due to the shift property of the Fourier transform, an additional exponential factor $e^{-i k v}$ appears under the integral. Integration with respect to $v$ gives the Fourier transform of $h(v)$ with a reciprocal velocity $k \tau$. The Fourier transform of (40) is:

$$
u_{k}(t)=\int_{0}^{t} u_{k}(t-\tau) h_{k \tau} f(\tau) d \tau+n_{0, k} \delta(t)
$$

where the indices $k$ and $k \tau$ denote the Fourier components. Note that,

$$
\mathcal{F}\left\{h(v)=\int_{-\infty}^{\infty} e^{-i k \tau v} h(v) d v\right\}=h(k \tau)
$$

. Next, we apply the Laplace transform with respect to time and use its convolution property to obtain the following:

$$
u_{k, s}=u_{k, s}\left[h_{k \tau} f(\tau)\right]_{s}+n_{0, k}
$$

where the index $s$ corresponds to the Laplace component. So the path traversed in search can be given in the Fourier-Laplace domain, $k, s$, as:

$$
u_{k, s}=\frac{n_{0, k}}{1-\left[h_{k \tau} f(\tau)\right]_{s}}
$$

We can introduce the result in (44) into the Fourier-Laplace expression for equation (41) (similar to above) to get the analytic expression for the density of trades in the Fourier-Laplace domain, obtained through the search process. 


$$
\begin{gathered}
q_{k}(t)=\int_{0}^{t} \frac{u_{k}(t-\tau) h_{k \tau} f(\tau)}{\eta_{k}(\tau)} d \tau \\
q_{k, s}=\frac{u_{k, s}\left[h_{k \tau} f(\tau)\right]_{s}}{\eta_{k, s}} \\
q_{k, s}=\frac{n_{0, k}}{\eta_{k, s}} \frac{\left[h_{k \tau} f(\tau)\right]_{s}}{\left(1-\left[h_{k \tau} f(\tau)\right]_{s}\right)}
\end{gathered}
$$

To find a solution, the next step is to take the Laplace inverse of equation (47). However, an analytic representation and direct inversion of the equation (47) is not feasible. Froemberg et al. (2015) recommend an asymptotic analysis for large space and time scales, $x, t \rightarrow \infty$. We use the same approach. Going to Fourier-laplace space using the tauberian theorem, this limit corresponds to $(k, s) \rightarrow(0,0)$ such that $k / s=$ constant. This has to be performed numerically.

It is important to define the velocity and flight time distributions before we move to obtain the inverse transformation. In our view this needs to come from empirical analysis. Further, velocity distribution cannot be obtained directly as it is a notional quantity and needs to be interpreted from equation (39) that describes the relationship with returns.

As discussed in Zaburdaev et al. (2008), Froemberg et al.(2015) and Zaburdaev et al. (2015) when the velocity distribution is Cauchy or lorentian, the density of the particles also is lorentian independent of flight times and jump lengths. Such a lorentian velocity profile appears in real physical phenomena such as two dimensional turbulence and is also found in model distributions of kinetic theory, statistics, plasma physics and starving amoeba cells. We know that a cautchy process does not give rise to a continuous sample path for the price and it differs from Brownian motion as there are large jumps not infrequently. As given below we make arbitrary choice of a lorentian velocity distribution $h(v)$ and intuitively a flight time distribution $f(\tau)$ with power tails.

$$
\begin{gathered}
h(v)=\frac{1}{u_{0} \pi} \frac{1}{\left(1+\left(\frac{v^{2}}{u_{0}^{2}}\right)\right)} \\
f(\tau)=\frac{\gamma}{(1+\tau)^{1+\gamma}}
\end{gathered}
$$

In equation (48) $u_{0}$ is needed to constrain the velocities so that the particles do not go beyond the ballistic cones, else it will lead to instantaneous dispersion. The $\gamma$ in equation (49) is varied to get different transport. $\gamma=1 / 2$ for normal diffusion. In equation (47), given the asymptotic limit we want to evaluate, we further set 
a constant efficiency, so that there is an expression for the initial density of particles for the trade density. The propagator for our model can then be noted as follows:

$$
G(k, s)=\frac{\mathcal{L}[f(\tau) h(k \tau)]}{1-\mathcal{L}[f(\tau) h(k \tau)]}
$$

where $k \tau$ is the Fourier variable conjugate to $v$. The equation (50) retains the form of the well known MontrollWeiss equation for the pdf of the uncoupled continuous time random walk (CTRW) to find the particle $x$ at the time $t$, modified such that it applies to random jumps in velocity. Equation (50) can be rewritten as (see Appendix C for details):

$$
G(k, s)=\frac{\left.\int_{-\infty}^{\infty} d v f(s+i k v \tau) h(v)\right]}{\left.1-\int_{-\infty}^{\infty} d v f(s+i k v \tau) h(v)\right]}
$$

For the flight time distribution we have chosen and in the long time limit the expansion in the Laplace space is given by,

$$
f(\tau) \approx 1-\tau^{\gamma} \Gamma(1-\gamma) s^{\gamma}
$$

Using (52) the asymptotic version of (51) is,

$$
G(k, s)=\frac{1}{s} \frac{\int_{-\infty}^{\infty}(1+i k v / s)^{\gamma-1} h(v) d v}{\int_{-\infty}^{\infty}(1+i k v / s)^{\gamma} h(v) d v}
$$

\section{B Inversion of the fourier laplace expression for propagators with ballis- tic scaling}

A method exists for the inversion of the fourier laplace expression for propagators with ballistic scaling. A propagator of a random walk model has ballistic scaling if it can be written in the form, $G(x, t) \approx \frac{1}{t} \phi\left(\frac{x}{t}\right), t \rightarrow$ $\infty$, where $\phi$ is the scaling function. In Fourier-laplace space this is, $G(k, s) \approx \frac{1}{s} g\left(\frac{i k}{s}\right)$. Comparing the above two forms we can rewrite the scaling form of our equation as in equation (54), where $\xi=\frac{i k}{s}$.

$$
g(\xi)=\frac{\int_{-\infty}^{\infty}(1+\xi v)^{\gamma-1} h(v) d v}{\int_{-\infty}^{\infty}(1+\xi v)^{\gamma} h(v) d v}
$$

Further, this can be inverted according to the general equation (55) (from Froemberg et al. (2015) based on Godreche and Luck (2001)) to obtain equation (56). The scaling function in (55) is defined as $\phi(y)=\langle\delta(y-Y)\rangle$. Here angular brackets denote the averaging with respect to a random variable $X$ which has a pdf $P(X),\langle F(X)\rangle=$ $\int_{-\infty}^{\infty} F(X) P(X) d X$ and $Y$ is the time average of the particles velocity, i.e $x / t$. (55) is obtained using Sokhotsky- 
Weirstrass theorem: $\frac{\lim }{\epsilon \rightarrow 0} \frac{1}{x \pm i \epsilon}=\frac{1}{x} \mp i \pi \delta(x)$ and thus $\mp \frac{1}{\pi} \operatorname{Im} \frac{\lim }{\epsilon \rightarrow 0} \frac{1}{x \pm i \epsilon}=\delta(x)$.

$$
\begin{gathered}
\phi(y)=\frac{-1}{\pi} \frac{\lim }{\epsilon \rightarrow 0} \mathfrak{J}\left[\frac{1}{y+i \epsilon} g\left(\frac{-1}{y+i \epsilon}\right)\right] \\
\phi(y)=\frac{-1}{\pi} \frac{\lim }{\epsilon \rightarrow 0} \mathfrak{I}\left[\frac{\int_{-\infty}^{\infty}(1+i \epsilon-v)^{\gamma-1} h(v) d v}{\int_{-\infty}^{\infty}(1+i \epsilon-v)^{\gamma} h(v) d v}\right]
\end{gathered}
$$

While the velocity distribution could be anything from a two state, or uniform distribution (Froemberg et al. (2015) discuss a number of examples) we arbitrarily choose the special situation induced by Cauchy distributed velocity. To use this we start with the following propagator and velocity distribution. The propagator reduces to (59) without prescribing to any particular form for $f(\tau)$.

$$
\begin{gathered}
G(k, s)=\frac{\{f(\tau) h(k \tau)\}}{1-\{f(\tau) h(k \tau)\}} \\
h(k \tau)=\exp \left(-u_{0}|k| \tau\right) \\
G(k, s)=\frac{1}{s+u_{0}|k|}
\end{gathered}
$$

Now taking the inverse Laplace and Fourier transform we get a form of Cauchy distribution. The scaling function is $\phi(y)=\frac{1}{\pi\left(1+y^{2}\right)}$.

$$
G(x, t)=\frac{u_{0} t}{\pi\left(u_{0}^{2} t^{2}+x^{2}\right)}
$$

\section{Explanation for equation (9)}

Numerator,

$$
\begin{gathered}
\mathcal{L}[f(\tau) h(k \tau)]_{(k, s)} \\
\left.=\int_{-\infty}^{\infty} d \tau \int_{-\infty}^{\infty} e^{-i k \tau v} e^{-s \tau} f(\tau) h(v)\right] \\
\left.=\int_{-\infty}^{\infty} d \tau \int_{-\infty}^{\infty} e^{-(s+i k v) \tau} e^{-s \tau} f(\tau) h(v)\right]
\end{gathered}
$$




$$
\left.=\int_{-\infty}^{\infty} d \tau \int_{-\infty}^{\infty} f(s+i k v) h(v)\right]
$$

Similarly the denominator can be arrived at.

\section{Search for trades in presence of bias (The Complete Model)}

We build a comprehensive model of stochastic evolution of market particles. Our fundamental set up of the complete model is (61) which is expanded to (62)

$$
\begin{gathered}
\frac{\partial g(x, t)}{\partial t}= \\
\frac{\partial}{\partial x}\left(D(t) \frac{\partial g(x, t)}{\partial x}-\lambda_{1}(t) \frac{\partial f(x, t)}{\partial x} g(x, t)-\lambda_{2}(t) g(x, t)\right)+v(t) g(x, t)+q(x, t) \\
\frac{\partial g(x, t)}{\partial t}=D(t) \frac{\partial^{2} g(x, t)}{\partial x^{2}}-\lambda_{1}(t) \frac{\partial f(x, t)}{\partial x} \frac{\partial g(x, t)}{\partial x}-\lambda_{1}(t) \frac{\partial^{2} f(x, t)}{\partial x^{2}} g(x, t) \\
-\lambda_{2}(t) \frac{\partial g(x, t)}{\partial x}+v(t) g(x, t)+q(x, t)
\end{gathered}
$$

(62) can be solved analytically. We draw upon the technique used by Sanskrityayn and Kumar (2016), who used the Greens function method to solve their diffusion-advection equation in the context of pollutant solutes in the atmosphere. To solve the equation, we note that $q$ will remain untouched and we need to reduce the equation to a known form so that we find an expression for $f(x, t)$. We do a co-ordinate transformation from the domain $(x, t)$ to the domain $\left(X(x, t), t^{\prime}\right)$. The domain $\mathrm{X}$ is essentially fixed time-snapshots of the entire lattice. We want to transform (62) to the form in equation (63).

$$
\left.\frac{\partial G\left(X, t^{\prime}\right)}{\partial t^{\prime}}=D_{1}\left(t^{\prime}\right) \frac{\partial^{2} G\left(X, t^{\prime}\right)}{\partial X^{2}}-\lambda\left(t^{\prime}\right) \frac{\partial G\left(X, t^{\prime}\right)}{\partial X}+v_{1}\left(t^{\prime}\right) G\left(X, t^{\prime}\right)\right)+q_{1}\left(X, t^{\prime}\right)
$$

Using the domain transformation, we can write equation (62) as equation (64), following which we equate the coefficients to obtain equation (65,66 and 67):

$$
\frac{\partial G\left(X, t^{\prime}\right)}{\partial t^{\prime}}=D\left(t^{\prime}\right)\left(\frac{\partial X}{\partial x}\right)^{2} \frac{\partial^{2} G\left(X, t^{\prime}\right)}{\partial X^{2}}
$$




$$
\begin{gathered}
-\left[-D\left(t^{\prime}\right) \frac{\partial^{2} X}{\partial x^{2}}+\lambda_{1}\left(t^{\prime}\right) \frac{\partial f(x, t)}{\partial x} \frac{\partial X}{\partial x}+\lambda_{2}(t) \frac{\partial X}{\partial x}+\frac{\partial X}{\partial t}\right] \frac{\partial G\left(X, t^{\prime}\right)}{\partial X} \\
\left.+\left[v\left(t^{\prime}\right)-\lambda_{1}\left(t^{\prime}\right) \frac{\partial f^{2}(x, t)}{\partial x^{2}}\right] G\left(X, t^{\prime}\right)\right)+q\left(X, t^{\prime}\right) \\
D\left(t^{\prime}\right)\left(\frac{\partial X}{\partial x}\right)^{2}=D_{1}(t) \\
-\left[-D\left(t^{\prime}\right) \frac{\partial^{2} X}{\partial x^{2}}+\lambda_{1}\left(t^{\prime}\right) \frac{\partial f(x, t)}{\partial x} \frac{\partial X}{\partial x}+\lambda_{2}(t) \frac{\partial X}{\partial x}+\frac{\partial X}{\partial t}\right]=-\lambda \\
v\left(t^{\prime}\right)-\lambda_{1}\left(t^{\prime}\right) \frac{\partial f^{2}(x, t)}{\partial x^{2}}=v_{1}
\end{gathered}
$$

From (65) we obtain an expression for $X$ in (68) and from (67) an expression for $f$ in (69). We insert these two results into (66) and equate similar coefficients to obtain (70) and (71). For the sake of convenience, we use t instead of $t^{\prime}$ hence forth.

$$
\begin{gathered}
X=\sqrt{\frac{D_{1}}{D}} x+\phi_{1}(t) \\
f(x, t)=\frac{v(t)-v_{1}(t)}{\lambda_{1}(t)} x^{2}+\phi_{2}(t) x+c \\
-\lambda_{1}(t)\left(\frac{v(t)-v_{1}(t)}{\lambda_{1}(t)} x+\phi_{2}(t)\right) \sqrt{\frac{D_{1}(t)}{D(t)}}-\lambda_{2}(t) \sqrt{\frac{D_{1}(t)}{D(t)}}-\frac{\partial}{\partial t}\left(\sqrt{\frac{D_{1}(t)}{D(t)}} x+\phi_{1}\right) \\
=-\lambda(t) \quad \\
\frac{\partial}{\partial t} \phi_{1}(t)-\lambda_{2}(t) \sqrt{\frac{D_{1}(t)}{D(t)}}-\lambda_{1}(t) \phi_{2}(t) \sqrt{\frac{D_{1}(t)}{D(t)}}=-\lambda(t) \\
-\left(v(t)-v_{1}(t)\right) \sqrt{\frac{D_{1}(t)}{D(t)}}=\frac{\partial}{\partial t} \sqrt{\frac{D_{1}(t)}{D(t)}}
\end{gathered}
$$


From (72), we get (73) where $\beta$ is a dimensionless expression defined in (74)

$$
\begin{gathered}
\frac{D_{1}(t)}{D(t)}=\frac{1}{\beta^{2}(t)} \\
\beta=e^{\int_{0}^{t}\left(v(s)-v_{1}(s)\right) d s}
\end{gathered}
$$

Using the expression for $\phi_{1}(t)$, obtained after reorganising (71), we obtain the expression for $\mathrm{X}$ as below,

$$
X=\frac{x}{\beta(t)}+\int_{0}^{t}\left(\lambda(t)-\frac{\lambda_{2}(t)}{\beta(t)}-\lambda_{2}(t) \frac{\phi_{2}(t)}{\beta(t)}\right)
$$

Equipped with the expression we have obtained for $f(x, t)$ in (69) and the transformations $\beta(t)$ and $X$ in (75) we can reduce our initial equation to the following form,

$$
\frac{\partial G(X, t)}{\partial t}=\frac{D(t)}{\beta^{2}(t)} \frac{\partial^{2} G(X, t)}{\partial X^{2}}-\lambda(t) \frac{\partial G(X, t)}{\partial X}+v_{1} G(X, t)+q_{1}(X, t)
$$

The initial conditions for this equation are $G(X, 0)=G_{i} \omega(X)$, with $-\infty<X<\infty$ and $t>0$. Next, we try to remove the drift term and the decay term. We use the following transformation equations one after the other for this purpose. In (78) $\beta_{1}=e^{\int_{0}^{t} v_{1}(s) d s}$ is a dimensionless term and in (79) $\beta$ is as per (73). In (79) T is a time variable. Equation (76) now reduces to equation (80).

$$
\begin{gathered}
\eta=X-\lambda(t) t \\
K(\eta, t)=\frac{G(\eta, t)}{\beta_{1}(t)} \\
T=\int_{0}^{t} \frac{1}{\beta^{2}(s)} d s \\
\frac{\partial K(\eta, T)}{\partial T}=D \frac{\partial^{2} K(\eta, T)}{\partial \eta^{2}}+\frac{Q(\eta, T) \beta^{2}(T)}{\beta_{1}}
\end{gathered}
$$

We now need to solve equation (80) to obtain a master equation for transport of market particles in double auction limit order asset markets. Haberman (1987) provides a solution for equations such as (80) using Greens Function Method (GFM). The solution to (80) based on the above is given in (81): 


$$
\begin{aligned}
K(\eta, T) & =\int_{0}^{T} \int_{-\infty}^{\infty} \frac{Q(\chi, \tau) \beta^{2}(\zeta)}{\sqrt{4 \pi D(T-\zeta)} \beta_{1}} \exp \left(-\frac{(\eta-\chi)^{2}}{4 D(T-\zeta)}\right) d \chi d \zeta \\
& +\int_{-\infty}^{\infty} \frac{1}{\sqrt{4 \pi D T}} G_{i} \omega(X) \exp \left(-\frac{(\eta-\chi)^{2}}{4 D T}\right) d \chi
\end{aligned}
$$

Next we sequentially trace back the transformations done earlier, in reverse order to get the solution below in (82).

Here, $\zeta=\int_{0}^{\tau} \frac{1}{\beta^{2}(s)} d s$ and the initial condition $g(x, 0)=G_{i} \omega(x)$.

$$
\begin{gathered}
g(x, t)=\beta_{1}(t) \int_{0}^{t} \int_{-\infty}^{\infty} \frac{Q(\chi, \tau)}{\sqrt{4 \pi D(T-\zeta)}} \exp \left(\frac{\left(\frac{\chi}{\beta}-\int_{0}^{t}\left(\frac{\lambda_{2}}{\beta}+\frac{\lambda_{1} \phi_{2}}{\beta}\right) d s-\chi\right)^{2}}{4 D(T-\zeta)}\right) d \chi d \tau \\
+\beta_{1}(t) \int_{-\infty}^{\infty} \frac{1}{\sqrt{4 \pi D T}} G_{i} \omega(\chi) \exp \left(-\frac{\left(\frac{\chi}{\beta}-\int_{0}^{t}\left(\frac{\lambda_{2}}{\beta}+\frac{\lambda_{1} \phi_{2}}{\beta}\right) d s-\chi\right)^{2}}{4 D T}\right) d \chi
\end{gathered}
$$

